\title{
Information Transmission using the Nonlinear Fourier Transform, Part I: Mathematical Tools
}

\author{
Mansoor I. Yousefi and Frank R. Kschischang, Fellow, IEEE
}

\begin{abstract}
The nonlinear Fourier transform (NFT), a powerful tool in soliton theory and exactly solvable models, is a method for solving integrable partial differential equations governing wave propagation in certain nonlinear media. The NFT decorrelates signal degrees-of-freedom in such models, in much the same way that the Fourier transform does for linear systems. In this threepart series of papers, this observation is exploited for data transmission over integrable channels such as optical fibers, where pulse propagation is governed by the nonlinear Schrödinger equation. In this transmission scheme, which can be viewed as a nonlinear analogue of orthogonal frequency-division multiplexing commonly used in linear channels, information is encoded in the nonlinear frequencies and their spectral amplitudes. Unlike most other fiber-optic transmission schemes, this technique deals with both dispersion and nonlinearity directly and unconditionally without the need for dispersion or nonlinearity compensation methods. This first paper explains the mathematical tools that underlie the method.
\end{abstract}

Index Terms-Nonlinear Fourier transform, integrable channels, Lax pairs, Zakharov-Shabat spectral problem, Fourier transforms, fiber-optic communications.

\section{INTRODUCTION}

A $\mathrm{N}$ EVOLUTION equation is a partial differential equation (PDE) for an unknown function $q(t, z)$ of the form

$$
q_{z}=K(q),
$$

where $K(q)$ is an expression involving $q$ and its derivatives with respect to $t$. In (1) and throughout this paper, subscripts are used to denote partial derivatives with respect to the corresponding variable.

The evolution equation (1) is a PDE in $1+1$ dimensions, i.e., one variable $t \in \mathbb{R}$ represents a temporal dimension and one variable $z \geqslant 0$ represents a spatial dimension. In most mathematical literature, the roles of $z$ and $t$ are interchanged, so rather than a spatial evolution as in (1), mathematicians study temporal evolution.

An important example of an evolution equation, and the one that motivates this work, is the stochastic nonlinear Schrödinger (NLS) equation governing the complex envelope

Submitted for publication on February 16, 2012; revised February 15, 2013; accepted March 21, 2013. The material in this paper was presented in part at the 2013 IEEE International Symposium on Information Theory. The authors are with the Edward S. Rogers Sr. Dept. of Electrical and Computer Engineering, University of Toronto, Toronto, ON M5S 3G4, Canada. Email: \{mansoor, frank\}@comm. utoronto.ca. of a narrowband signal in an optical fiber in the presence of noise, given by [1], [2]

$$
\begin{aligned}
\frac{\partial Q(\tau, \ell)}{\partial \ell}= & \frac{j \beta_{2}}{2} \frac{\partial^{2} Q(\tau, \ell)}{\partial \tau^{2}}-j \gamma Q(\tau, \ell)|Q(\tau, \ell)|^{2} \\
& +N(\tau, \ell), \quad 0 \leqslant \ell \leqslant \mathcal{L} .
\end{aligned}
$$

Here $\ell$ denotes distance (in $\mathrm{km}$ ) along the fiber; the transmitter is located at $\ell=0$, and the receiver is located at $\ell=\mathcal{L}$. The symbol $\tau$ represents retarded time, measured in seconds, i.e., $\tau=t-\beta_{1} \ell$ where $t$ is ordinary time and $\beta_{1}$ is a constant, and $Q(\tau, \ell)$ is the complex envelope of the signal propagating in the fiber. The coefficient $\beta_{2}$, measured in $\mathrm{s}^{2} / \mathrm{km}$, is called the chromatic dispersion coefficient, while $\gamma$, measured in $\mathrm{W}^{-1} \mathrm{~km}^{-1}$, is the nonlinearity parameter. Finally, $N(\tau, \ell)$ is bandlimited white Gaussian noise with in-band spectral density $\sigma_{0}^{2}(\mathrm{~W} /(\mathrm{km} \cdot \mathrm{Hz}))$ and autocorrelation

$$
\mathrm{E}\left\{N(\tau, \ell) N^{*}\left(\tau^{\prime}, \ell^{\prime}\right)\right\}=\sigma_{0}^{2} \delta_{W}\left(\tau-\tau^{\prime}\right) \delta\left(\ell-\ell^{\prime}\right),
$$

where $\delta_{W}(x)=2 W \operatorname{sinc}(2 W x)$. It is assumed that $Q(\tau, \ell)$ is bandlimited to $W$ for all $\ell, 0 \leqslant \ell \leqslant \mathcal{L}$.

The stochastic NLS equation (2) models both chromatic dispersion (captured by the $j \beta_{2} \partial^{2} Q / \partial \tau^{2}$ term), which is responsible for temporal broadening, and the Kerr nonlinearity (captured by the $\gamma|Q|^{2} Q$ term), which is responsible for spectral broadening. Pulse propagation is governed by the tension between these effects and can be linearly dominated, nonlinearly dominated, or solitonic (in which case the two effects are balanced). The NLS equation defines a nonlinear dispersive waveform channel from $Q(\tau, 0)$ at the transmitter to $Q(\tau, \mathcal{L})$ at the receiver.

Such a nonlinear dispersive waveform channel is a major departure from the classical additive white Gaussian noise and wireless fading channels in terms of analytical difficulty. Here the signal degrees-of-freedom couple together via the nonlinearity and dispersion in a complicated manner, making it difficult to establish the channel input-output map, even deterministically. Most current approaches assume a linearlydominated regime of operation, consider the nonlinearity as a small perturbation, or are geared towards managing and suppressing the (detrimental) effects of the nonlinear and dispersive terms. In-line dispersion management, digital backpropagation, and other forms of electronic pre-and postcompensation belong to this class of methods (see [3]-[7] and references therein). 
In this paper we adopt a different philosophy. Rather than treating nonlinearity and dispersion as nuisances, we seek a transmission scheme that is fundamentally compatible with these effects. We effectively "diagonalize" the nonlinear Schrödinger channel with the help of the nonlinear Fourier transform (NFT), a powerful tool for solving integrable nonlinear dispersive partial differential equations [8], [9]. The NFT uncovers linear structure hidden in the one-dimensional cubic nonlinear Schrödinger equation, and can be viewed as a generalization of the (ordinary) Fourier transform to certain nonlinear systems.

With the help of the nonlinear Fourier transform, we are able to represent a signal by its discrete and continuous nonlinear spectra. While the signal propagates along the fiber based on the complicated NLS equation, the action of the channel on its spectral components is given by simple independent linear equations. Just as the (ordinary) Fourier transform converts a linear convolutional channel $y(t)=x(t) * h(t)$ into a number of parallel scalar channels, the nonlinear Fourier transform converts a nonlinear dispersive channel described by a Lax convolution (see Sec. III) into a number of parallel scalar channels. This suggests that information can be encoded (in analogy with orthogonal frequency-division multiplexing) in the nonlinear spectra.

The nonlinear Fourier transform is intertwined with the existence of soliton solutions to the NLS equation. Solitons are pulses that retain their shape (or return periodically to their initial shape) during propagation, and can be viewed as system eigenfunctions, similar to the complex exponentials $e^{j \omega t}$, which are eigenfunctions of linear systems. An arbitrary waveform can be viewed as a combination of solitons, associated with the discrete nonlinear spectrum, and a non-solitonic (radiation) component, associated with the continuous nonlinear spectrum.

The goal of this first article is to introduce the mathematical tools that underlie this approach to information transmission. These tools are sufficiently general to encompass not only the nonlinear Schrödinger equation, but also other completely integrable nonlinear dispersive PDEs. Thus, the transmission scheme described here can also be applied to any channel model in this general class. These tools are also described in mathematics and physics (see, e.g., [8]-[10]); here we attempt to extract those aspects of the theory that are relevant to the engineering aspects of the information transmission problem. In Part II [11] we will provide numerical methods for forward NFT at the receiver and in Part III [12] describe a nonlinear frequency-division multiplexing communication method based on NFT, provide algorithms to implement inverse NFT at the transmitter and give examples of achievable spectral efficiencies in actual fiber-optic systems.

After submitting this paper, we became aware of the related paper of Hasegawa and $\mathrm{Nyu}$ [13] introducing eigenvalue communication, in which the authors propose to encode information in quantities conserved under NLS propagation, and use the inverse scattering transform to decode the amplitude of an isolated soliton-like pulse $A \operatorname{sech}(t)$ in a single-user, pointto-point channel. Using conserved quantities can be helpful, as it has the potential to simplify communication system design.
However, no improvement in capacity is achieved relative to a scheme which encodes information in an equivalent, but not necessarily conserved, set of parameters (e.g., modulating amplitude and phase and using backpropagation for decoding).

Our motivation for introducing transmission schemes based on the NFT stems - not from the simplicity arising through the use of conserved quantities (as in [13]) but rather-from our observation that the use of conventional linear multiplexing in nonlinear channels inevitably leads to an interference-limited multiuser system [12]. As a result, a major factor contributing to the capacity limitations in optical fiber networks [3] is the use of wavelength-division multiplexing. By introducing nonlinear frequency-division multiplexing (NFDM) based on the NFT (exploiting the integrability of the NLS equation), a transmission scheme without deterministic distortions can result; see [Part III, particularly Sections VI. F and VI. G] for further details about NFDM in comparison with [13]. Thus we use the tools of integrable systems for a different purpose than in [13].

We will find it convenient to work with the nonlinear Schrödinger equation (2) in a normalized form. By changing variables

$$
q=\frac{Q}{\sqrt{P}}, \quad z=\frac{l}{\mathcal{L}}, \quad t=\frac{\tau}{T_{0}},
$$

with $T_{0}=\sqrt{\left|\beta_{2}\right| \mathcal{L} / 2}$ and $P=2 /(\gamma \mathcal{L})$, we get the normalized NLS equation

$$
j q_{z}(t, z)=q_{t t}+2|q(t, z)|^{2} q(t, z)+n(t, z),
$$

where

$$
\mathrm{E}\left\{n(t, z) n^{*}\left(t^{\prime}, z^{\prime}\right)\right\}=\frac{\sigma_{0}^{2} \mathcal{L}}{P T_{0}} \delta_{W_{n}}\left(t-t^{\prime}\right) \delta\left(z-z^{\prime}\right),
$$

where $W_{n}=W T_{0}$ is the normalized bandwidth. Throughout this paper, (3) will be our primary and motivating illustrative example.

\section{A BRiEF History OF THE NONLINEAR FourieR TRANSFORM}

The nonlinear Fourier transform (also known as the inverse scattering transform or IST) was originally developed as a method for solving certain nonlinear dispersive partial differential equations. These are integrable PDEs, i.e., nonlinear differential equations exhibiting certain hidden linearity. There are several integrable equations having physical significance, among which is the NLS equation. The IST method was a result of extensive efforts in theoretical physics and applied mathematics in 1960s and later, closely associated with the notion of solitons in integrable models [8], [9].

In the 1950s, in one of the first dynamical-systems simulations performed on a computer [14], Fermi, Pasta and Ulam performed a numerical experiment to understand why solids have finite heat conductivity. They modeled the solid as a lattice with point masses at the lattice points coupled with springs each having a quadratic nonlinearity. To their surprise, rather than observing an equipartition of energy among all Fourier modes, energy cycled periodically among a 
few low-order modes. Such behavior implies that the nonlinear oscillator behaves somehow linearly.

In the 1960s, Zabusky and Kruskal showed that equation of motion for the Fermi-Pasta-Ulam lattice in the continuum limit is a remarkable PDE called the Korteweg-de Vries (KdV) equation [15], known in the study of water waves. The KdV equation for the evolution of a real-valued pulse $q(t, z)$ as a function of time $t$ and distance $z$ is

$$
q_{z}=q q_{t}+q_{t t t} .
$$

Zabusky and Kruskal found that (4) has pulse-like (localized) solutions whose shape is preserved (or varies periodically) during propagation. Furthermore, they made the surprising observation that when two such pulses are launched towards each other, despite their nonlinear interaction, they pass through each other without changing their shape. Zabusky and Kruskal coined the term soliton for such solutions, in recognition of their particle-like properties [15].

The spectacular properties of these solutions greatly excited the mathematics and physics communities and many researchers started to study solitons. In a celebrated paper [16], Gardner, Greene, Kruskal and Miura uncovered some of the deep structure underlying the $\mathrm{KdV}$ equation which is responsible for solitons and their unusual properties. The authors of [16] were studying the celebrated linear Schrödinger equation from quantum mechanics, given by

$$
\psi_{z}(t, z)=\psi_{t t}(t, z)+g(t, z) \psi(t, z),
$$

where $\psi(t, z)$ is the wave-function and $g(t, z)$ is an external potential. They found that if one takes the solution $q(t, z)$ of the KdV equation (4) as the external potential $g$ in (5), then the eigenvalues of the Schrödinger operator

$$
H=\frac{\partial^{2}}{\partial t^{2}}+g(t, z)
$$

remain invariant during the evolution in $z$ ! Based on this critical observation, they developed a method to recover the external potential $g(t, z)=q(t, z)$ by solving an inverse problem for (5). The method analytically predicts soliton solutions for the KdV equation, as observed earlier by Zabusky and Kruskal through numerical computations. They had in fact found the IST for the special case of the KdV equation.

It was not immediately clear if the method developed in [16] could be generalized to other nonlinear PDEs, since it is not obvious if there exists a certain auxiliary operator, like the Schrödinger operator $H$, whose eigenvalues are preserved during the evolution. In a landmark paper published in 1968 [17], Lax put the theory on a firm mathematical footing. In particular, he established the mathematical relationship between the auxiliary operators with invariant eigenvalues (now called Lax pairs) and the original nonlinear equation. Once a Lax pair for a nonlinear PDE is found, a method along the lines of [16] can be applied to solve that PDE.

Shortly afterwards, in 1972, Zakharov and Shabat found a Lax pair for the NLS equation in one spatial dimension [1], and thus established that this equation, too, could be solved in the same manner. Details of this method for the NLS equation were subsequently developed by Ablowitz and others (see
[8] and references therein), who also referred to this scheme as the "nonlinear Fourier transform". After these discoveries from the 1960s and 70s, research into solitons became an established area of research, lying at the intersection of applied mathematics and nonlinear physics.

Nonlinear PDEs solvable by the NFT are called integrable equations or exactly solvable models [18]. These are usually Hamiltonian systems having an infinite number of conserved quantities, and include the KdV, NLS, modified KdV, sineGordon equations, and the Toda lattice, among others [8], [9]. These equations all exhibit similar properties, including the existence of soliton solutions. The generation and processing of soliton signals, and the engineering of novel transmission systems that support their propagation, is described in [19].

\section{Canonical Lax Form for Exactly Solvable MODELS}

\section{A. Lax Pairs and Evolution Equations}

We wish to consider linear differential operators whose eigenvalues are invariant during an evolution [17]. More precisely, we consider such operators defined in terms of a signal $q(t, z)$ where the eigenvalues of the operator remain constant even as $q$ evolves (in $z$ ) according to some evolution equation.

To facilitate the discussion, it is useful to imagine a linear operator represented as a matrix; however, we must keep in mind that, when moving from finite-dimensional spaces to infinite-dimensional spaces (of e.g., functions and operators), some results do not carry over necessarily. The relevant properties of linear operators needed for this paper are reviewed in Appendix $\mathrm{A}$

Let $L(z)$ be a square matrix whose entries are functions of $z$. Clearly, the eigenvalues of this matrix are in general functions of $z$ too. However, for some matrices, it might be the case that while the entries of the matrix change with $z$, the eigenvalues remain constant (independent of $z$ ). Such a matrix, if diagonalizable, should be similar to a constant diagonal matrix $\Lambda$, i.e., $L(z)=G(z) \Lambda G^{-1}(z)$, for some similarity transformation $G(z)$.

This idea generalizes to operators. Let $\mathcal{H}$ be a Hilbert space, let $\mathcal{D}$ be some domain that is dense in $\mathcal{H}$, and let $L(z)$ : $\mathcal{D} \rightarrow \mathcal{H}$ be a family of bounded linear operators indexed by a parameter $z$ [20]. If the eigenvalues of $L(z)$ do not depend on $z$, then we refer to $L(z)$ as an isospectral family of operators. If diagonalizable, it follows that for each $z, L(z)$ is similar to a multiplication operator $\Lambda$ (the operator equivalent of a diagonal matrix; see Appendix $\mathrm{A})$, i.e., $L(z)=G(z) \Lambda G^{-1}(z)$, for some operator $G(z)$.

Assuming that $L(z)$ varies smoothly with $z$, we can consider the rate of the change (with respect to $z$ ) of $L(z)$. We have

$$
\begin{aligned}
\frac{\mathrm{d} L(z)}{\mathrm{d} z} & =G_{z} \Lambda G^{-1}+G \Lambda\left(-G^{-1} G_{z} G^{-1}\right) \\
& =G_{z} G^{-1}\left(G \Lambda G^{-1}\right)-\left(G \Lambda G^{-1}\right) G_{z} G^{-1} \\
& =M(z) L(z)-L(z) M(z)=[M, L],
\end{aligned}
$$

where $G_{z}=\mathrm{d} G(z) / \mathrm{d} z, M=G_{z} G^{-1}$, and $[M, L] \triangleq$ $M L-L M$ is the commutator bracket. In other words, every 


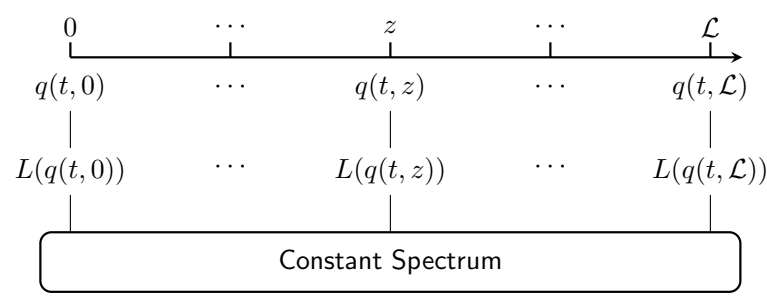

Fig. 1. An isospectral flow: the spectrum of $L$ is held invariant even as $q(t, z)$ evolves.

diagonalizable isospectral operator $L(z)$ satisfies the differential equation (7).

Conversely, suppose $M(z)$ is given and the (unknown) diagonalizable operator $L(z)$ evolves according to (7) with initial condition $L(0)=G_{0} \Lambda_{0} G_{0}^{-1}$. Let $G(z)$ be the (unique invertible) solution to $G_{z}=M G$ with $G(0)=G_{0}$. One can easily verify that $L(z)=G(z) \Lambda_{0} G(z)^{-1}$ satisfies (7). Assuming that the solution to a first-order differential equation is unique [21], we see that $L(z)$ is an isospectral family.

The characterization of isospectral operators is therefore summarized in the following lemma [17].

Lemma 1. Let $L(z)$ be a diagonalizable operator. Then $L(z)$ is isospectral if and only if it satisfies

$$
\frac{\mathrm{d} L}{\mathrm{~d} z}=[M, L],
$$

for some operator $M$. If $L$ is self-adjoint (so that $L$ is unitarily equivalent to a multiplication operator, i.e., $\left.L=G \Lambda G^{*}\right)$, then $M$ must be skew-Hermitian, i.e., $M^{*}=-M$.

Proof: The proof was outlined above. The skewHermitian property of $M$ can be shown by differentiating $G G^{*}=I$.

It is important to note that $L$ and $M$ do not have to be independent and can depend on a common parameter, e.g., a function $q(t, z)$, as illustrated in Fig. 1. The isospectral property of the solution is unchanged. The commutator bracket $[M, L]$ in (8) can create nonlinear evolution equations for $q(t, z)$ in the form

$$
\frac{\partial q}{\partial z}=K(q),
$$

where $K(q)$ is some, in general nonlinear, function of $q(t, z)$ and its time derivatives. An example of this is the KdV equation.

Example 1 (KdV equation). Let $D=\frac{\partial}{\partial t}$ denote the timederivative operator, and let $q(t, z)$ be a real-valued function. Finally, let

$$
\begin{aligned}
L & =D^{2}+q, \text { and let } \\
M & =\frac{3}{2} D^{3}+\frac{1}{2}(D q+q D) .
\end{aligned}
$$

The Lax equation $L_{z}=[M, L]$ is easily simplified to

$$
q_{z}-\left(q_{t t t}+q q_{t}\right)+(\text { some terms }) D \equiv \mathbf{0},
$$

where $\mathbf{0}$ is the zero operator. The zero-order term of this equation as a polynomial in $D$, which must be zero, produces the KdV equation $q_{z}=q_{t t t}+q q_{t}$. Using $\left(D^{k}\right)^{*}=(-1)^{k} D$ (provable by integration by parts), it is easy to see that $L$ and $M$ are self-adjoint and skew-Hermitian, respectively, since they are real-valued and involve even and odd powers of $D$, respectively. The eigenvalues of $L$ are thus preserved from Lemma 1 Note that the $L$ operator in this example is precisely the (linear) Schrödinger operator $H$ given in (6).

Definition 1. A pair of operators $L$ and $M$, depending on $z$, are called a Lax pair $(L, M)$ if they satisfy the Lax equation (8). Following Lemma 1, the eigenvalues of the $L$ operator are independent of $z$.

\section{B. The Zero-Curvature Condition}

The eigenvalues of the operator $L$, which are constant in an isospectral flow, are defined via

$$
L v=\lambda v .
$$

Taking the $z$ derivative of (9) and using the Lax equation $L_{z}=$ $[M, L]$, we obtain $(L-\lambda I)\left(v_{z}-M v\right)=0$. Since $L-\lambda I$ vanishes only on eigenvectors of $L$, it must be that $v_{z}-M v$ is an element of the eigenspace, i.e., $v_{z}-M v=\alpha G x, \alpha \in \mathbb{C}$, and $x$ is a coordinate vector. The choice of $\alpha$ does not influence the results of future sections; thus for simplicity we set $\alpha=0$. It follows that an eigenvector $v(t, z)$ evolves based on the linear equation

$$
v_{z}=M v .
$$

Furthermore, it is often more convenient to re-write (9) as

$$
v_{t}=P v,
$$

for some operator $P$. The relationship between $P$ and $L$ can be derived (if necessary) by combining $(D I-P) v=0$ with $(L-\lambda I) v=0$, obtaining

$$
P=\Sigma(L-\lambda I)+D I,
$$

where $\Sigma$ is some invertible operator, and $D=\frac{\partial}{\partial t}$ as in Example 1

Combining equations (10) and (11) by using the equality of mixed derivatives, i.e., $v_{t z}=v_{z t}$, the Lax equation (8) is reduced to the zero-curvature condition [9]

$$
P_{z}-M_{t}+[P, M]=0 .
$$

Note that the nonlinear equation derived from (13) results as a compatibility condition between the two linear equations (10) and (11). This shows that certain nonlinear equations possess a "hidden linearity" in the form of (10) and (11).

Following the work of Zakharov and Shabat on the NLS equation [1], Ablowitz et al. [22] suggested that for many equations of practical significance, the operator $P$ can be fixed as

$$
P=\left(\begin{array}{cc}
-j \lambda & r(t, z) \\
s(t, z) & j \lambda
\end{array}\right),
$$

where $r(t, z)$ and $s(t, z)$ are functions-depending on $q(t, z)$ - to be determined to produce a given nonlinear evolution equation. From (12) this corresponds to the $L$ operator

$$
L=j\left(\begin{array}{cc}
D & -r(t, z) \\
s(t, z) & -D
\end{array}\right),
$$


with $\Sigma=\operatorname{diag}(j,-j)$. In this case, both $L$ and $P$ operate on $2 \times 1$ vector functions.

Equation (11), with $P$ as in (14), is known as the AKNS system (after the authors of [22]) and is central in the study of the nonlinear Fourier transform [9]. The important special case where $r(t, z)=q(t, z)$ and $s(t, z)=-q^{*}(t, z)$ is generally known as the Zakharov-Shabat system. We will refer to (9), (10) and (11) as the $L-, M$-, and $P$-equations, respectively. Throughout this paper, we will assume that the domain of $L$, $P$, and $M$ are subsets of the Hilbert space $L^{2}(\mathbb{R})$, denoted here by $\mathcal{H}$, depending on the particular structure of that operator.

Example 2 (Sine-Gordon equations). Let $r(t, z)=-s(t, z)=$ $\frac{1}{2} q_{t}(t, z)$ in (14) and let

$$
M=\frac{j}{4 \lambda}\left(\begin{array}{cc}
\cos (q) & -\sin (q) \\
-\sin (q) & -\cos (q)
\end{array}\right) .
$$

Then the zero-curvature equation is simplified to $q_{t z}=\sin (q)$. Taking $r=s=\frac{1}{2} q_{t}$ and $M$ as

$$
M=\frac{j}{4 \lambda}\left(\begin{array}{cc}
\cosh (q) & -\sinh (q) \\
\sinh (q) & -\cosh (q)
\end{array}\right)
$$

gives $q_{t z}=\sinh (q)$.

Example 3 (Nonlinear Schrödinger equation). Take $r=q$, $s=-q^{*}$ and

$$
M=\left(\begin{array}{cc}
2 j \lambda^{2}-j|q(t, z)|^{2} & -2 \lambda q(t, z)-j q_{t}(t, z) \\
2 \lambda q^{*}(t, z)-j q_{t}^{*}(t, z) & -2 j \lambda^{2}+j|q(t, z)|^{2}
\end{array}\right) .
$$

The zero-curvature equation is simplified to $j q_{z}(t, z)=$ $q_{t t}(t, z)+2|q(t, z)|^{2} q(t, z)$.

Example $4\left(\mathrm{KdV}\right.$ equation revisited). Let $r=\frac{2}{\sqrt{3}} q, s=$ $-\frac{1}{4 \sqrt{3}}$ and

$M=\left(\begin{array}{cc}4 j \lambda^{3}-\frac{j \lambda q}{3}+\frac{q_{t}}{6} & -\frac{8 \lambda^{2} q}{\sqrt{3}}-\frac{4}{\sqrt{3}} j \lambda q_{t}+\frac{2 q^{2}}{3 \sqrt{3}}+\frac{2 q_{t t}}{\sqrt{3}} \\ \frac{\lambda^{2}}{\sqrt{3}}-\frac{q}{12 \sqrt{3}} & -4 j \lambda^{3}+\frac{j \lambda q}{3}-\frac{q_{t}}{6}\end{array}\right)$.

The zero-curvature equation leads to $q_{z}=q q_{t}+q_{t t t}$.

It should be noted from Examples 1 and 4 that the choice of $L$ and $M$ giving rise to a given nonlinear equation is not unique. Obviously one can scale $L$ by a number, or add a constant $\alpha I$ to $L$ or $M$. In addition, both the Lax equation (8) and (9) are unchanged under orthogonal transformations, i.e., replacing $L$ and $M$ with $\Sigma L \Sigma^{T}$ and $\Sigma M \Sigma^{T}$, respectively, where $\Sigma$ is a (constant) orthogonal matrix, i.e., $\Sigma^{T} \Sigma=I$. Note further that it may be possible to choose two Lax pairs $\left(L_{1}, M_{1}\right)$ and $\left(L_{2}, M_{2}\right)$ for a given equation such that $L_{1}$ is self-adjoint and $L_{2}$ is not self-adjoint. The eigenvalues of $L_{1}$ and $L_{2}$ are, respectively, real and complex; see Appendix A

\section{Lax Convolution and Integrable Communication Channels}

Linear systems traditionally have been described by linear constant coefficient differential equations. An example is the one-dimensional heat equation $q_{z}=c^{2} q_{t t}$, where $c$ is the diffusion coefficient and $q(t, z)$ represents the heat profile across a rod extending in space $t$, as time $z$ goes on. From a systems point of view, this defines a linear time-invariant

$$
x(t) \stackrel{=q(t, 0)}{\longrightarrow} L_{z}(q)=[M(q), L(q)] \stackrel{y(t)=q(t, \mathcal{L})}{\longrightarrow}
$$

Fig. 2. A system defined by Lax convolution.

system from input $x(t)=q(t, 0)$ at $z=0$ to the output $y(t)=q(t, \mathcal{L})$ at some $z=\mathcal{L}$. The role of $z$ is therefore just a parameter and once fixed (to $z=\mathcal{L}$ ), the system is described by an impulse response (or a Green function) $h(t ; \mathcal{L})$, representing the underlying (linear) convolution.

Following this analogy, we wish to define a system in terms of a Lax pair $(L, M)$. Here, $L$ and $M$ are parametrized by a waveform $q(t, z)$. Such a system accepts a waveform $x(t)=$ $q(t, 0)$ at its input and produces a waveform $y(t)=q(t, \mathcal{L})$ at its output, according to the evolution equation induced by $L_{z}=[M, L]$. The time-domain input-output map is thus given by an evolution equation of the form $q_{z}=K(q)$, obtainable from the Lax equation (8) (or its equivalent (13)). We refer to such a system as an integrable system. Note that an integrable system is completely characterized by the two operators $(L, M)$ and the parameter $z=\mathcal{L}$, independent of the signals. We denote such a system using the triple $(L, M ; \mathcal{L})$.

Definition 2 (Lax convolution). We refer to the action of an integrable system $S=(L, M ; \mathcal{L})$ on the input $q(t, 0)$ as the Lax convolution of $q$ with $S$. We write the system output as $q(t, \mathcal{L})=q(t, 0) *(L, M ; \mathcal{L})$. See Fig. 2 .

Definition 3 (Integrable communication channels). A waveform communication channel $C: x(t) \times n(t, z) \rightarrow y(t)$ with inputs $x(t) \in L^{1}(\mathbb{R})$ and space-time noise $n(t, z) \in$ $L^{1}\left(\mathbb{R}, \mathbb{R}^{+}\right)$, and output $y(t) \in L^{1}(\mathbb{R})$, is said to be integrable if the noise-free channel is an integrable system.

Note that noise can be introduced into an integrable system in a variety of ways. In the approach taken in these papers, we assume that the signal-to-noise ratio is not too small, so that the stochastic system may justifiably be considered as a perturbation of the deterministic system. Furthermore, we limit ourselves to integrable channels with additive noise, i.e.,

$$
q_{z}=K(q)+n(t, z), \quad q(t, 0)=x(t), \quad q(t, \mathcal{L})=y(t),
$$

where $n(t, z)$ is distributed band-limited noise. Note that this model is not in general equivalent to one in which noise is added (in lumped fashion) at the channel output. Here, the noise is distributed in space, and so interacts with the signal (in a potentially complicated manner) according to the given evolution equation.

In this paper, we develop a scheme for communication over integrable channels. By various choices of Lax pair $(L, M)$, one can construct a variety of interesting channel models, mostly nonlinear, which go beyond the linear channel models typically studied in data communications. Interestingly, some linear channels can also be analyzed using this nonlinear spectral approach advocated here [23]. As noted in Section \, the central application (and motivation) for this work is fiberoptic communication, in which the channel model is given by the nonlinear Schrödinger equation (2), and for which a Lax 
pair was given in Example 3. In this paper we first discuss the deterministic (noise-free) case and later, in [Part III], treat noise as a perturbation of the deterministic system.

\section{NONLINEAR Fourier TRANSFORM}

In this section, we assume that a function $q(t, \cdot)$ is given, and we define its nonlinear Fourier transform with respect to the Lax operator $L(15)$. As the notation $q(t, \cdot)$ implies, in this and the next section, the variable $z$ can take on any value in the range $[0, \mathcal{L}]$ and is irrelevant in the forward and inverse transforms. We shall therefore omit the index $z$ in what follows.

Assumptions 1. We assume that $q(t)$ satisfies:

1) $q(t) \in L^{1}(\mathbb{R})$;

2) $q(t) \rightarrow 0$ as $|t| \rightarrow \infty$.

As previously noted, for concreteness we carry through the development of the NFT for the case of the NLS equation (3), for which the $P$-equation is the Zakharov-Shabat system

$$
v_{t}=P(\lambda, q) v=\left(\begin{array}{cc}
-j \lambda & q(t) \\
-q^{*}(t) & j \lambda
\end{array}\right) v .
$$

If for a given $\lambda \in \mathbb{C}$ the operator $L-\lambda I$ is not invertible, then we say that $\lambda$ belongs to the spectrum of $L$ and $v(t, \lambda)$ represents its associated eigenvector. In finite-dimensional Hilbert spaces of matrices the spectrum is a discrete finite set of eigenvalues. This may no longer be true in infinitedimensional spaces of operators, where the eigenvalues (if they exist) may only be one part of the spectrum. See Appendix A

The nonlinear Fourier transform of a signal $x(t)$ with respect to an operator $L$ in a Lax pair is defined via the spectral analysis of the $L$ operator, which we consider next.

\section{A. Canonical Eigenvectors and Spectral Coefficients}

We wish to study solutions of (16), in which vectors $v(t)=\left(v_{1}(t), v_{2}(t)\right)^{T}$ are considered as elements of the vector space $\mathcal{H}$. We begin by equipping the vector space $\mathcal{H}$ with a symplectic bilinear form $\mathcal{H} \times \mathcal{H} \mapsto \mathbb{C}$, which, for any fixed value of $t \in \mathbb{R}$, is defined as

$$
\langle v(t), w(t)\rangle_{s}=v_{1}(t) w_{2}(t)-v_{2}(t) w_{1}(t) .
$$

Let us also define the adjoint of any vector $v$ in $\mathcal{H}$ as

$$
\tilde{v}(t)=\left(\begin{array}{r}
v_{2}^{*}(t) \\
-v_{1}^{*}(t)
\end{array}\right) .
$$

The following properties hold true for all $v$ and $w$ in $\mathcal{H}$ :

- $\tilde{\tilde{v}}=-v$;

- $\langle v, v\rangle_{s}=0$

- $\langle v, w\rangle_{s}=-\langle w, v\rangle_{s}$

- $\langle\tilde{v}, v\rangle_{s}=-\langle v, \tilde{v}\rangle_{s}=\left|v_{1}\right|^{2}+\left|v_{2}\right|^{2}$;

- $\frac{\mathrm{d}}{\mathrm{d} t}\langle v, w\rangle_{s}=\left\langle v_{t}, w\right\rangle_{s}+\left\langle v, w_{t}\right\rangle_{s}$;

- for every $2 \times 2$ matrix $A,\langle A v, w\rangle_{s}+\langle v, A w\rangle_{s}=$ $\operatorname{tr}(A)\langle v, w\rangle_{s}$.

There are generally infinitely many solutions $v$ of (16) for a given $\lambda \in \mathbb{C}$, parametrized by the set of all possible boundary conditions. These solutions form a subspace $E_{\lambda}$ of continuously differentiable $2 \times 1$ vector functions (an eigenspace).

Lemma 2. For all vectors $v(t)$ and $w(t)$ in $E_{\lambda}$,

1) $\tilde{v} \in E_{\lambda^{*}}$, i.e., $\tilde{v}_{t}=P\left(\lambda^{*}, q\right) \tilde{v}$;

2) $\langle v(t), w(t)\rangle_{s}$ is a constant, independent of $t$;

3) If $\langle v(t), w(t)\rangle_{s} \neq 0$, then $v$ and $w$ are linearly independent and form a basis for $E_{\lambda}$;

4) $\operatorname{dim}\left(E_{\lambda}\right)=2$.

Proof: Property 10 follows directly from (16). To see 2), note that $\frac{\mathrm{d}}{\mathrm{d} t}\langle v, w\rangle_{s}=\left\langle v_{t}, w\right\rangle_{s}+\left\langle v, w_{t}\right\rangle_{s}=\langle P v, w\rangle_{s}+$ $\langle v, P w\rangle_{s}=\operatorname{tr}(P)\langle v, w\rangle_{s}=0$. To see 3), fix $t$ and let $u(t) \in E_{\lambda}$, then $u(t)=a(t) v(t)+b(t) w(t)$ for some $a(t)$ and $b(t)$. Taking the symplectic inner product of both sides with $w$ and $v$, we get $a(t)=\langle u, w\rangle_{s} /\langle v, w\rangle_{s}$ and $b(t)=\langle u, v\rangle_{s} /\langle w, v\rangle_{s}$. From Property $2\langle u, w\rangle_{s},\langle v, w\rangle_{s}$, $\langle u, v\rangle_{s}$, and $\langle w, v\rangle_{s}$ are all independent of $t$. It follows that $a$ and $b$ are also independent of $t$. Finally, 4) follows from 3).

An important conclusion of Lemma 2 is that any two linearly independent solutions $u$ and $w$ of (16) provide a basis for the solution space. To choose two such solutions, we examine the behavior of the equation at large values of $|t|$. By Assumption 112, as $|t| \rightarrow \infty$ (16) is reduced to

$$
v_{t} \rightarrow\left(\begin{array}{cc}
-j \lambda & 0 \\
0 & j \lambda
\end{array}\right) v, \quad \text { for large }|t|,
$$

which has a general solution

$$
v(t, \lambda) \rightarrow\left(\alpha e^{-j \lambda t}, \beta e^{j \lambda t}\right)^{T}, \quad \alpha, \beta \in \mathbb{C} .
$$

Two possible boundary conditions, bounded in the upper half complex plane $\mathbb{C}^{+}=\{\lambda: \Im(\lambda)>0\}$, are

$$
\begin{aligned}
& v^{1}(t, \lambda) \rightarrow\left(\begin{array}{l}
0 \\
1
\end{array}\right) e^{j \lambda t}, \quad t \rightarrow+\infty, \\
& v^{2}(t, \lambda) \rightarrow\left(\begin{array}{l}
1 \\
0
\end{array}\right) e^{-j \lambda t}, \quad t \rightarrow-\infty .
\end{aligned}
$$

We solve (16) for a given $\lambda \in \mathbb{C}^{+}$under the boundary conditions (17a $-(17 \mathrm{~b})$, and denote the resulting solutions for all $t \in \mathbb{R}$ as $v^{1}(t, \lambda)$ and $v^{2}(t, \lambda)$. We can also solve (16) for $\lambda^{*}$ under the adjoint boundary conditions, bounded in the lower half complex plane $\mathbb{C}^{-}=\{\lambda: \Im(\lambda)<0\}$,

$$
\begin{array}{ll}
v^{1}\left(t, \lambda^{*}\right) & \rightarrow\left(\begin{array}{l}
0 \\
1
\end{array}\right) e^{j \lambda^{*} t}, \quad t \rightarrow+\infty, \\
v^{2}\left(t, \lambda^{*}\right) & \rightarrow\left(\begin{array}{c}
-1 \\
0
\end{array}\right) e^{-j \lambda^{*} t}, \quad t \rightarrow-\infty,
\end{array}
$$

giving rise to two solutions $v^{1}\left(t, \lambda^{*}\right)$ and $v^{2}\left(t, \lambda^{*}\right)$. Taking tilde " $\sim$ " operation, we obtain $\tilde{v}^{1}\left(t, \lambda^{*}\right)$ and $\tilde{v}^{2}\left(t, \lambda^{*}\right)$. From Lemma 2 we have that $\tilde{v}^{1}\left(t, \lambda^{*}\right)$ and $\tilde{v}^{2}\left(t, \lambda^{*}\right)$ are elements of $E_{\lambda}$. These four eigenvectors $v^{1}(t, \lambda), v^{2}(t, \lambda), \tilde{v}^{1}\left(t, \lambda^{*}\right)$, and $\tilde{v}^{2}\left(t, \lambda^{*}\right)$, all of them elements of $E_{\lambda}$, are called canonical eigenvectors. Fig. 3 illustrates the canonical eigenvectors at their boundaries.

Lemma 3. Canonical eigenvectors satisfy:

1) $\left\langle\tilde{v}^{1}\left(t, \lambda^{*}\right), v^{1}(t, \lambda\rangle_{s}=\left\langle v^{2}(t, \lambda), \tilde{v}^{2}\left(t, \lambda^{*}\right)\right\rangle_{s}=1\right.$; 


$$
\begin{array}{cc}
v^{2}(t, \lambda) \rightarrow\left(\begin{array}{c}
1 \\
0
\end{array}\right) e^{-j \lambda t} & v^{1}(t, \lambda) \\
t=-\infty & \rightarrow\left(\begin{array}{l}
0 \\
1
\end{array}\right) e^{j \lambda t} \\
v^{2}\left(t, \lambda^{*}\right) \rightarrow\left(\begin{array}{c}
-1 \\
0
\end{array}\right) e^{-j \lambda^{*} t} & t=\infty \\
\tilde{v}^{2}\left(t, \lambda^{*}\right) & v^{1}\left(t, \lambda^{*}\right) \rightarrow\left(\begin{array}{l}
0 \\
1
\end{array}\right) e^{j \lambda^{*} t} \\
\tilde{v}^{1}\left(t, \lambda^{*}\right)
\end{array}
$$

Fig. 3. Boundary conditions for the canonical eigenvectors.

2) $\left\{v^{1}(t, \lambda), \tilde{v}^{1}\left(t, \lambda^{*}\right)\right\}$ and $\left\{v^{2}(t, \lambda), \tilde{v}^{2}\left(t, \lambda^{*}\right)\right\}$ are independent sets in $E_{\lambda}$;

3) $\overline{v^{1}(t, \lambda)}=\tilde{v}^{1}\left(t, \lambda^{*}\right)$ and $\widetilde{v^{2}(t, \lambda)}=-\tilde{v}^{2}\left(t, \lambda^{*}\right)$.

Proof: 1) Since $\left\langle\tilde{v}^{1}, v^{1}\right\rangle_{s}$ is independent of $t$, using (17a) and (18a), $\left\langle\tilde{v}^{1}\left(t, \lambda^{*}\right), v^{1}(t, \lambda)\right\rangle_{s}=$ $\left\langle\tilde{v}^{1}\left(+\infty, \lambda^{*}\right), v^{1}(+\infty, \lambda)\right\rangle_{s}=1$. 2) Follows from 1) and Lemma 2 3) All four canonical eigenvectors are in $E_{\lambda}$. This part thus follows by noting the boundary conditions (17a)-18b).

Choosing $\tilde{v}^{1}\left(t, \lambda^{*}\right)$ and $v^{1}(t, \lambda)$ as a basis of $E_{\lambda}$, one can project $v^{2}(t, \lambda), \tilde{v}^{2}\left(t, \lambda^{*}\right) \in E_{\lambda}$ on this basis to obtain

$$
\begin{aligned}
v^{2}(t, \lambda) & =a(\lambda) \tilde{v}^{1}\left(t, \lambda^{*}\right)+b(\lambda) v^{1}(t, \lambda), \\
\tilde{v}^{2}\left(t, \lambda^{*}\right) & =-b^{*}\left(\lambda^{*}\right) \tilde{v}^{1}\left(t, \lambda^{*}\right)+a^{*}\left(\lambda^{*}\right) v^{1}(t, \lambda),
\end{aligned}
$$

where $a(\lambda)=\left\langle v^{2}, v^{1}\right\rangle_{s}, b(\lambda)=\left\langle\tilde{v}^{1}, v^{2}\right\rangle_{s}$, and the coefficients in (19b) are obtained by applying the tilde " $\sim$ " operation to (19a). A crucial property, following from Lemma 2, is that $a(\lambda)$ and $b(\lambda)$ are time-independent. The time-independent complex scalars $a(\lambda)$ and $b(\lambda)$ are called the nonlinear Fourier coefficients [8], [9].

Since the nonlinear Fourier coefficients are time independent, to facilitate computing them, for simplicity we can send $t$ to, e.g., $+\infty$ where $v^{1}(+\infty, \lambda)$ and $\tilde{v}^{1}\left(+\infty, \lambda^{*}\right)$ are known. The other two canonical eigenvectors $v^{2}$ and $\tilde{v}^{2}$ are then propagated from their boundary values $v^{2}(-\infty, \lambda)$ and $\tilde{v}^{2}\left(-\infty, \lambda^{*}\right)$ at $t=-\infty$ according to $v_{t}^{2}=P(\lambda, q) v^{2}$ and $\tilde{v}_{t}^{2}=P\left(\lambda^{*}, q\right) \tilde{v}^{2}$ to obtain $v^{2}(\infty, \lambda)$ and $\tilde{v}^{2}\left(\infty, \lambda^{*}\right)$. At this stage, we have available all four canonical eigenvectors at one time, namely,

$$
\left\{v^{2}(+\infty, \lambda), \tilde{v}^{2}\left(+\infty, \lambda^{*}\right), v^{1}(+\infty, \lambda), \tilde{v}^{1}\left(+\infty, \lambda^{*}\right)\right\} .
$$

We can now project $v^{2}(+\infty, \lambda)$ and $\tilde{v}^{2}\left(+\infty, \lambda^{*}\right)$ onto the basis $v^{1}(+\infty, \lambda)$ and $\tilde{v}^{1}\left(+\infty, \lambda^{*}\right)$ according to 19a $-19 \mathrm{~b}$ to obtain

$$
\left(v^{2}(+\infty, \lambda), \tilde{v}^{2}\left(+\infty, \lambda^{*}\right)\right)=\left(\tilde{v}^{1}\left(+\infty, \lambda^{*}\right), v^{1}(+\infty, \lambda)\right) S,
$$

where

$$
S=\left(\begin{array}{cc}
a(\lambda) & -b^{*}\left(\lambda^{*}\right) \\
b(\lambda) & a^{*}\left(\lambda^{*}\right)
\end{array}\right) .
$$

The matrix $S$ is called the scattering matrix and contains the nonlinear Fourier coefficients [8], [9]. It is a function of $q(t, \cdot)$ and says how the solution to (16) is scattered from $t=-\infty$ to $t=+\infty$. More precisely, the field $v^{2}(-\infty, \lambda)=$ $(1,0)^{T} e^{-j \lambda t}$ is applied at $t=-\infty$, where $q$ is absent. This field evolves forward in time according to (16), interacts with the signal (which can be viewed as an "obstacle") at finite values of $t$, and subsequently propagates towards $t=+\infty$, where again $q$ is absent. The field at $t=+\infty$ is measured and gives information about the "obstacle" as seen from a distance. Although not obvious from the development so far, we shall see in Section VII that the information measured at $t=+\infty$, captured by $a(\lambda)$ and $b(\lambda)$, is complete, in the sense that from this information we can retrieve $q(t, \cdot)$ entirely. In view of this interpretation, the nonlinear Fourier transform was historically referred to as the inverse scattering transform.

Note that, taking the determinant of the both sides of (20), $\operatorname{det} S=a(\lambda) a^{*}\left(\lambda^{*}\right)+b(\lambda) b^{*}\left(\lambda^{*}\right)=1$.

\section{B. The Nonlinear Fourier Transform}

The projection equations (19a) and (19b) that give $a(\lambda)$ and $b(\lambda)$ are well-defined if $\lambda \in \mathbb{R}$. From Lemma 2, Property 1), we observe that the eigenspace is symmetric in $\lambda$, i.e., if $\lambda$ is an eigenvalue then so is $\lambda^{*}$. Thus it is sufficient to consider the upper half complex plane $\mathbb{C}^{+}$. In this region, the boundary conditions on the basis vectors $v^{1}$ and $\tilde{v}^{1}$ at $t=\infty$ decay and blow up, respectively. As a result, $19 \mathrm{a}$ is consistent in $\mathbb{C}^{+}$ only if $a(\lambda)=0$. Eigenvalues in $\mathbb{C}^{+}$are therefore identified as the zeros of the complex function $a(\lambda)$ and they form the discrete (point) spectrum of the signal. We will see in the next section that the discrete spectrum corresponds to soliton pulses.

Lemma 4. If $q(t) \in L^{1}(\mathbb{R}), a(\lambda)$ is an analytic function of $\lambda$ on $\mathbb{C}^{+}$.

Proof: From Lemma 5 in Section VII if $q(t) \in L^{1}(\mathbb{R})$ the scaled canonical eigenvectors $v^{1}(t, \lambda) e^{-j \lambda t}$ and $v^{2}(t, \lambda) e^{j \lambda t}$ are analytic functions of $\lambda$ in $\mathbb{C}^{+}$. Since

$$
a(\lambda)=\left\langle v^{2}, v^{1}\right\rangle_{s}=\left\langle v^{2} e^{j \lambda t}, v^{1} e^{-j \lambda t}\right\rangle_{s},
$$

is a combination of two analytic functions in $\mathbb{C}^{+}$, it is analytic in the same region. (Note, however, that $b(\lambda)$, which is a combination of functions analytic in disjoint regions in $\mathbb{C}$, may not be analytic in either of those regions.)

A consequence of Lemma 4 is that the zeros of $a(\lambda)$ in $\mathbb{C}^{+}$are isolated points [24]. It follows that the ZakharovShabat operator for the NLS equation has two types of spectra. The discrete (or point) spectrum, which occurs in $\mathbb{C}^{+}$, is characterized by those $\lambda_{j} \in \mathbb{C}^{+}$satisfying

$$
a\left(\lambda_{j}\right)=0, j=1,2, \ldots, N .
$$

The discrete spectrum corresponds to solitons, and in this case (19a) reduces to

$$
v^{2}\left(t, \lambda_{j}\right)=b\left(\lambda_{j}\right) v^{1}\left(t, \lambda_{j}\right) .
$$

The continuous spectrum, which in general includes the whole real line $\Im(\lambda)=0$, corresponds to the non-solitonic (or radiation) component of the signal. The continuous spectrum is the component of the NFT which corresponds to the ordinary Fourier transform, whereas the discrete spectrum has no analogue in linear systems theory. The reader is referred to Appendix $\mathrm{A}$ for a number of examples illustrating 
various notions of the spectrum associated with bounded linear operators.

To distinguish between the discrete and continuous spectra, we find it convenient to refer to discrete spectral values of $\lambda$ using the symbol $\lambda_{j}$ (with a subscript). Continuous spectral values are denoted as $\lambda$ (without a subscript). In general, $\lambda_{j} \in$ $\mathbb{C}^{+}$, whereas $\lambda \in \mathbb{R}$.

For the purpose of developing the inverse transform, we find it sufficient to work with the ratios

$$
\hat{q}(\lambda)=\frac{b(\lambda)}{a(\lambda)}, \quad \tilde{q}\left(\lambda_{j}\right)=\frac{b\left(\lambda_{j}\right)}{a_{\lambda}\left(\lambda_{j}\right)},
$$

where $a_{\lambda}\left(\lambda_{j}\right)$ denotes the derivative $\mathrm{d} a(\lambda) /\left.\mathrm{d} \lambda\right|_{\lambda=\lambda_{j}}$.

We can now formally define the nonlinear Fourier transform of a signal with respect to the Lax operator $L$ (15) as follows.

Definition 4 (Nonlinear Fourier transform [8], [10]). Let $q(t)$ be a sufficiently smooth function satisfying Assumptions 1 The nonlinear Fourier transform of $q(t)$ with respect to the Lax operator $L$ (15) consists of the continuous and discrete spectral functions $\hat{q}(\lambda): \mathbb{R} \mapsto \mathbb{C}$ and $\tilde{q}\left(\lambda_{j}\right): \mathbb{C}^{+} \mapsto \mathbb{C}$ where

$$
\hat{q}(\lambda)=\frac{b(\lambda)}{a(\lambda)}, \quad \tilde{q}\left(\lambda_{j}\right)=\frac{b\left(\lambda_{j}\right)}{a_{\lambda}\left(\lambda_{j}\right)}, \quad j=1,2, \ldots, N,
$$

in which $\lambda_{j}$ are the zeros of $a(\lambda)$. Here, the spectral coefficients $a(\lambda)$ and $b(\lambda)$ are given by

$$
\begin{aligned}
& a(\lambda)=\lim _{t \rightarrow \infty} v_{1}^{2} e^{j \lambda t}, \\
& b(\lambda)=\lim _{t \rightarrow \infty} v_{2}^{2} e^{-j \lambda t},
\end{aligned}
$$

where $v^{2}(t, \lambda)$ is a solution of (16) under the boundary condition $17 \mathrm{~b})$.

To obtain the continuous spectral function $\hat{q}(\lambda), \lambda \in \mathbb{R}$, it is not necessary to find $a(\lambda)$ and $b(\lambda)$ separately. For convenience, one can instead write an explicit differential equation for $y(t, \lambda)=\left(v_{2}^{2} / v_{1}^{2}\right) \exp (-2 j \lambda t)$ :

$$
\left\{\begin{array}{l}
\frac{\mathrm{d} y(t, \lambda)}{\mathrm{d} t}+q(t) e^{2 j \lambda t} y^{2}(t, \lambda)+q^{*}(t) e^{-2 j \lambda t}=0 \\
y(-\infty, \lambda)=0
\end{array}\right.
$$

and obtain $\hat{q}(\lambda)=\lim _{t \rightarrow \infty} y(t, \lambda)$.

Analogously, following (21a), one can solve the secondorder differential equation

$$
\left\{\begin{array}{l}
\frac{\mathrm{d}^{2} z(t, \lambda)}{\mathrm{d} t^{2}}-\left(2 j \lambda+\frac{q_{t}}{q}\right) \frac{\mathrm{d} z(t, \lambda)}{\mathrm{d} t}+|q|^{2} z(t, \lambda)=0 \\
z(-\infty, \lambda)=1, \quad \frac{\mathrm{d} z(-\infty, \lambda)}{\mathrm{d} t}=0
\end{array}\right.
$$

and obtain $a(\lambda)=\lim _{t \rightarrow \infty} z(t, \lambda)$. The discrete spectrum is obtained as the zeros of $a(\lambda)$.

Just like the ordinary Fourier transform, the nonlinear Fourier transform can be computed analytically only in a few cases. An example is given in the next subsection.

\section{Example: Nonlinear Fourier Transform of a Rectangular Pulse}

Consider the rectangular pulse

$$
q(t)= \begin{cases}A, & t \in\left[t_{1}, t_{2}\right] \\ 0, & \text { otherwise }\end{cases}
$$

Let $T=t_{2}-t_{1}$ and $T^{\prime}=t_{2}+t_{1}$.

In this case $P(\lambda, q)$ is time-independent when $t \in\left[t_{1}, t_{2}\right]$, and 16 under the boundary condition (17b) can be easily solved in closed form. The canonical eigenvector $v^{2}$ is given by

$v^{2}(t, \lambda)=\exp \left[\left(t-t_{1}\right) P\right] v^{2}\left(t_{1}, \lambda\right), \quad v^{2}\left(t_{1}, \lambda\right)=\left(\begin{array}{l}1 \\ 0\end{array}\right) e^{-j \lambda t_{1}}$.

It follows that

$$
v^{2}(\infty, \lambda)=v^{2}\left(t_{2}, \lambda\right)=\exp (P T) v^{2}\left(t_{1}, \lambda\right)
$$

where

$$
\begin{aligned}
& \exp (P T)=\exp \left\{\left(\begin{array}{cc}
-j \lambda & q \\
-q^{*} & j \lambda
\end{array}\right) T\right\} \\
& \quad=\left(\begin{array}{cc}
\cos (\Delta T)-j \frac{\lambda}{\Delta} \sin (\Delta T) & \frac{A}{\Delta} \sin (\Delta T) \\
\frac{-A^{*}}{\Delta} \sin (\Delta T) & \cos (\Delta T)+j \frac{\lambda}{\Delta} \sin (\Delta T)
\end{array}\right),
\end{aligned}
$$

with $\Delta=\sqrt{\lambda^{2}+|A|^{2}}$. The spectral coefficients are obtained from (21a) and (21b) as

$$
\begin{aligned}
& a(\lambda)=\left(\cos (\Delta T)-j \frac{\lambda}{\Delta} \sin (\Delta T)\right) e^{j \lambda T}, \\
& b(\lambda)=\frac{-A^{*}}{\Delta} \sin (\Delta T) e^{-j \lambda T^{\prime}} .
\end{aligned}
$$

The zeros of $a(\lambda)$ in $\mathbb{C}^{+}$, which satisfy

$$
j \tan \left(T \sqrt{|A|^{2}+\lambda^{2}}\right)=\sqrt{1+\frac{|A|^{2}}{\lambda^{2}}},
$$

give rise to the discrete spectrum. The continuous spectrum is given by

$$
\hat{q}(\lambda)=\frac{A^{*}}{j \lambda} e^{-2 j \lambda t_{2}}\left(1-\frac{\Delta}{j \lambda} \cot (\Delta T)\right)^{-1} .
$$

Note that as $A \rightarrow 0, \Delta \rightarrow \lambda$, and one can see that in the limit of $A T \ll 1$ there is no discrete spectrum. Furthermore, the continuous spectrum tends to

$$
\hat{q}(\lambda)=-A^{*} T e^{-j 2 \pi f T^{\prime}} \operatorname{sinc}(2 T f), \quad \lambda=2 \pi f,
$$

which is just the ordinary Fourier transform of the $-q^{*}(t)$ with $f \rightarrow 2 f$.

Fig. 4 shows the two spectra for $T=1$ and various values of $A$. For small $A$, there is no discrete spectrum and the continuous spectrum is essentially just the ordinary Fourier transform of $q(t)$. As $A$ is increased, the continuous spectrum deviates from the ordinary Fourier transform and one or more discrete mass points appear on the $j \omega$ axis. 


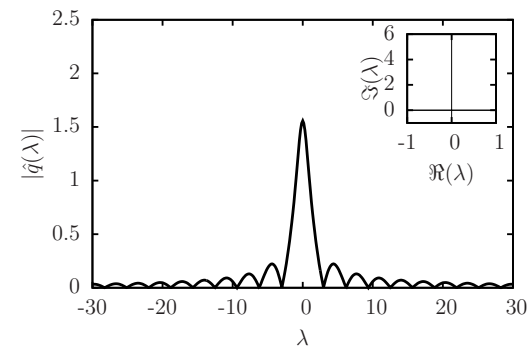

(a)

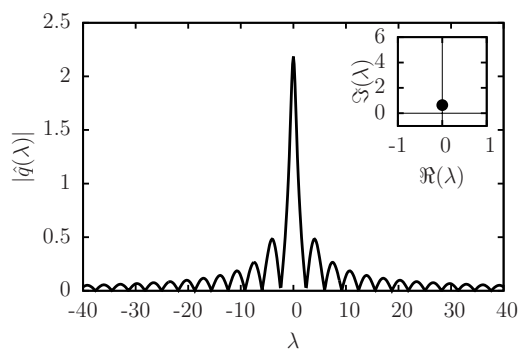

(b)

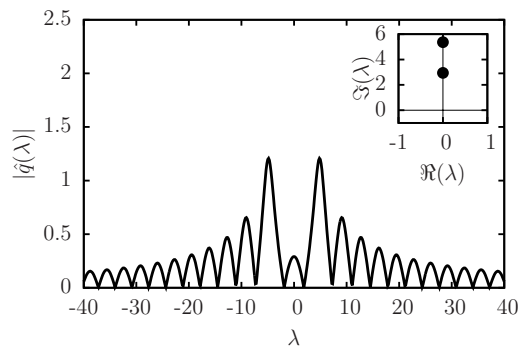

(c)

Fig. 4. Discrete and continuous spectra of the square wave signal with $T=1$ and (a) $A=1$, (b) $A=2$, (c) $A=6$.

\section{Elementary Properties of the Nonlinear Fourier Transform}

Let $q(t) \leftrightarrow\left(\widehat{q}(\lambda), \widetilde{q}\left(\lambda_{k}\right)\right)$ be a nonlinear Fourier transform pair. The following properties are proved in Appendix B

1) (The ordinary Fourier transform as limit of the nonlinear Fourier transform): If $\|q\|_{L^{1}} \ll 1$, there is no discrete spectrum and $\widehat{q}(\lambda) \rightarrow Q(\lambda)$, where $Q(\lambda)$ is the ordinary (linear) Fourier transform of $-q^{*}(t)$

$$
Q(\lambda)=-\int_{-\infty}^{\infty} q^{*}(t) e^{-2 j \lambda t} \mathrm{~d} t
$$

2) (Weak nonlinearity): If $|a| \ll 1$, then $\widehat{a q}(\lambda) \approx a \widehat{q}(\lambda)$. In general, however, $a q(t) \leftrightarrow a\left(\hat{q}(\lambda), \tilde{q}\left(\lambda_{k}\right)\right)$;

3) (Constant phase change): $e^{j \phi} q(t) \leftrightarrow e^{-j \phi}\left(\hat{q}(\lambda), \tilde{q}\left(\lambda_{k}\right)\right)$;

4) (Time dilation): $q\left(\frac{t}{a}\right) \leftrightarrow\left(\widehat{|a| q}(a \lambda), \widetilde{|a| q}\left(a \lambda_{k}\right)\right)$;

5) (Time shift): $q\left(t-t_{0}\right) \leftrightarrow e^{-2 j \lambda t_{0}}\left(\widehat{q}(\lambda), \widetilde{q}\left(\lambda_{k}\right)\right)$;

6) (Frequency shift): $q(t) e^{-2 j \omega t} \leftrightarrow\left(\widehat{q}(\lambda-\omega), \widetilde{q}\left(\lambda_{k}-\omega\right)\right)$;

7) (Lax convolution): If $q_{2}(t)=q_{1}(t) *(L, M ; \mathcal{L})$, then $\widehat{q_{2}}(\lambda)=H(\lambda, \mathcal{L}) \widehat{q_{1}}(\lambda)$ and $\widetilde{q_{2}}\left(\lambda_{k}\right)=H\left(\lambda_{k}, \mathcal{L}\right) \widetilde{q_{1}}\left(\lambda_{k}\right)$. For the NLS equation, the channel filter is $H(\lambda, \mathcal{L})=$ $\exp \left(-4 j \lambda^{2} \mathcal{L}\right)$

8) (Parseval's identity): $\int_{-\infty}^{\infty}\|q(t)\|^{2} \mathrm{~d} t=\hat{E}+\tilde{E}$, where

$$
\hat{E}=\frac{1}{\pi} \int_{-\infty}^{\infty} \log \left(1+|\hat{q}(\lambda)|^{2}\right) \mathrm{d} \lambda, \quad \tilde{E}=4 \sum_{j=1}^{N} \Im\left(\lambda_{j}\right) .
$$

The quantities $\hat{E}$ and $\tilde{E}$ represent the energy contained in the continuous and discrete spectra, respectively.

In addition, we have the following properties related to the nonlinear Fourier transform:

1) (Causality and layer-peeling property): Let $q_{1}(t)$ and $q_{2}(t)$ be two signals with non-overlapping support, e.g., signals in a pulse train. Without loss of generality, assume that $q_{1}(t)$ is supported on $t \leqslant t_{0}$, and that $q_{2}(t)$ is supported on $t>t_{0}$. If $\left(a_{1}, b_{1}\right)$ and $\left(a_{2}, b_{2}\right)$ are, respectively, the nonlinear Fourier coefficients of $q_{1}(t)$ and $q_{2}(t)$, then the nonlinear Fourier coefficients of $q_{1}(t)+q_{2}(t)$ are given by

$$
\begin{aligned}
(a(\lambda), b(\lambda))= & \left(a_{1}(\lambda), b_{1}(\lambda)\right) \circ\left(a_{2}(\lambda), b_{2}(\lambda)\right) \\
\triangleq & \left(a_{1}(\lambda) a_{2}(\lambda)-b_{1}(\lambda) b_{2}^{*}\left(\lambda^{*}\right),\right. \\
& \left.a_{1}(\lambda) b_{2}(\lambda)+b_{1}(\lambda) a_{2}^{*}\left(\lambda^{*}\right)\right) .
\end{aligned}
$$

That is to say, if we slice the signal in time in consecutive portions according to a mesh $-\infty<\cdots<t_{-1}<$ $t_{0}<t_{1}<\cdots<\infty$, the nonlinear Fourier coefficients satisfy the Markov property

$$
\begin{aligned}
\left(a_{k}(\lambda), b_{k}(\lambda) ; q(t), t \in\left(t_{k}, t_{k+1}\right)\right) & \\
& \rightarrow\left(a_{k+1}(\lambda), b_{k+1}(\lambda)\right),
\end{aligned}
$$

where $\left(a_{k}(\lambda), b_{k}(\lambda)\right)$ are the spectral coefficients calculated from $q(t)$ in $-\infty<t<t_{k}$

2) (Purely imaginary eigenvalues) If $\|q\|_{L^{1}}<\frac{\pi}{2}$, then there is no discrete spectrum. If $q(t) \in L^{1}(\mathbb{R})$ is real, non-negative, piece-wise smooth, and single-lobe (nondecreasing for $t<t_{0}$ and non-increasing for $t>t_{0}$ ), then there are precisely $N=\left\lfloor\frac{1}{2}+\frac{\|q(t)\|_{L^{1}}}{\pi}-\epsilon\right\rfloor$ eigenvalues, all purely imaginary and simple [25].

\section{Evolution OF THE NONLINEAR FOURIER TRANSFORM}

Derivation of the evolution of the nonlinear Fourier transform of a signal propagating based on the NLS equation proceeds straightforwardly. As $q(t, z)$ propagates, the eigenvalues of $L$ are preserved and the eigenvectors of $L$ propagate based on

$$
\begin{aligned}
v_{z} & =M(\lambda, q) v \\
& =\left(\begin{array}{cc}
2 j \lambda^{2}-j|q(t, z)|^{2} & -2 \lambda q(t, z)-j q_{t}(t, z) \\
2 \lambda q^{*}(t, z)-j q_{t}^{*}(t, z) & -2 j \lambda^{2}+j|q(t, z)|^{2}
\end{array}\right) v .
\end{aligned}
$$

Assuming that $q(t, z)$ and its time-derivative vanish at $t=$ $\pm \infty$ for all $z \leqslant \mathcal{L}$ during the propagation, then as $t \rightarrow \infty$ (24) is reduced to

$$
v_{z}(t, z) \rightarrow\left(\begin{array}{cc}
2 j \lambda^{2} & 0 \\
0 & -2 j \lambda^{2}
\end{array}\right) v(t, z) .
$$

Thus the boundary conditions $(17 \mathrm{a})$ and $(17 \mathrm{~b})$ are transformed to

$$
\begin{aligned}
& v^{1}(t, \lambda) \rightarrow\left(\begin{array}{l}
0 \\
1
\end{array}\right) e^{j \lambda t} e^{-2 j \lambda^{2} z}, \quad t \rightarrow+\infty \\
& v^{2}(t, \lambda) \rightarrow\left(\begin{array}{l}
1 \\
0
\end{array}\right) e^{-j \lambda t} e^{2 j \lambda^{2} z}, \quad t \rightarrow-\infty .
\end{aligned}
$$

These transformed boundary conditions are not consistent with the boundary conditions 17a and 17b used to define the canonical eigenvectors, due to the additional factors $e^{ \pm 2 j \lambda^{2} z}$. As a result, the evolution of the canonical eigenvectors from 
$z=0$, according to $v_{z}=M v$, does not lead to the canonical eigenvectors at $z=\mathcal{L}$. However, by proper scaling, one can obtain the canonical eigenvectors at any $z$.

For instance, focusing on $v^{2}(t, \lambda ; z)$, and changing variables to $u^{2}(t, \lambda ; z)=v^{2}(t, \lambda ; z) e^{-2 j \lambda^{2} z}$, we obtain $u_{t}^{2}=P u^{2}$ with boundary condition (17b) (at $t=-\infty)$ for all $z$. Consequently, $u^{2}(t, \lambda ; z)$ is a canonical eigenvector for all $z$. By transforming (25), the evolution equation for $u$ is asymptotically (at $t=$ $+\infty)$ given by

$$
u_{z}^{2}(\infty, \lambda ; z)=\left(\begin{array}{cc}
0 & 0 \\
0 & -4 j \lambda^{2} z
\end{array}\right) u^{2}(\infty, \lambda ; z),
$$

which gives

$$
\begin{aligned}
& u_{1}^{2}(\infty, \lambda ; z)=u_{1}^{2}(\infty, \lambda ; 0), \\
& u_{2}^{2}(\infty, \lambda ; z)=u_{2}^{2}(\infty, \lambda ; 0) e^{-4 j \lambda^{2} z} .
\end{aligned}
$$

Using expressions (21a) and (21b) we obtain

$$
\begin{aligned}
a(\lambda, z) & =\lim _{t \rightarrow \infty} u_{1}^{2}(t, \lambda ; z) e^{j \lambda t}=\lim _{t \rightarrow \infty} u_{1}^{2}(t, \lambda ; 0) e^{j \lambda t}=a(\lambda, 0), \\
b(\lambda, z) & =\lim _{t \rightarrow \infty} u_{2}^{2}(t, \lambda ; z) e^{-j \lambda t}=\lim _{t \rightarrow \infty} u_{2}^{2}(t, \lambda ; 0) e^{-4 j \lambda^{2} z} e^{-j \lambda t} \\
& =b(\lambda, 0) e^{-4 j \lambda^{2} z} .
\end{aligned}
$$

In turn, the nonlinear Fourier transform propagates according to

$$
\begin{aligned}
\widehat{q(t, z)}(\lambda) & =e^{-4 j \lambda^{2} z} \widehat{q(t, 0)}(\lambda), \\
\widehat{q(t, z)}\left(\lambda_{j}\right) & =e^{-4 j \lambda_{j}^{2} z} \overline{q(t, 0)}\left(\lambda_{j}\right), \\
\lambda_{j}(z) & =\lambda_{j}(0), \quad j=1,2, \ldots, N .
\end{aligned}
$$

Note that, since $a(\lambda, z)$ is preserved under the evolution (i.e., is independent of $z$ ), the number of the discrete eigenvalueswhich are zeros of $a(\lambda)$-is also preserved.

In summary, we see that the operation of the Lax convolution in the nonlinear Fourier domain is described by a simple multiplication (diagonal) operator, much in the same way that the ordinary Fourier transform maps $y(t)=x(t) * h(t)$ to $Y(\omega)=X(\omega) \cdot H(\omega)$. The channel filter (transfer function) in (26) is $\exp \left(-4 j \lambda^{2} z\right)$.

\section{AN APPROACH TO COMMUNICATION OVER INTEGRABLE CHANNELS}

Since the nonlinear Fourier transform of a signal is essentially preserved under Lax convolution, one can immediately conceive of a nonlinear analogue of orthogonal frequencydivision multiplexing (OFDM) for communication over integrable channels. We refer to this scheme as nonlinear frequency-division multiplexing. In this scheme, the inputoutput channel model is given by

$$
\begin{aligned}
\hat{Y}(\lambda) & =H(\lambda) \hat{X}(\lambda)+\hat{Z}, \\
\tilde{Y}\left(\lambda_{j}\right) & =H\left(\lambda_{j}\right) \tilde{X}\left(\lambda_{j}\right)+\tilde{Z}_{j},
\end{aligned}
$$

where $\hat{X}(\lambda)=\hat{q}(\lambda, 0)$ and $\tilde{X}\left(\lambda_{j}\right)=\tilde{q}\left(\lambda_{j}, 0\right)$ are spectra at the input of the channel, $\hat{Y}(\lambda)=\hat{q}(\lambda, z)$ and $\tilde{Y}\left(\lambda_{j}\right)=\tilde{q}\left(\lambda_{j}, z\right)$ are spectra at the output of the channel, and the channel filter is

$$
H(\lambda)=e^{-4 j \lambda^{2} z} .
$$

Here $\hat{Z}$ and $\tilde{Z}_{j}$ are effective noise in the spectral domain. Bandlimited Gaussian noise in the time domain generally transforms to non-Gaussian noise in the spectral domain. The noise variables affecting distinct eigenvalues are in general correlated and a function of the entire signal spectrum. Exact statistical description of the noise in the spectral domain is in general involved; however when noise is small a perturbation approach can easily be taken [Part III].

The proposed scheme consists of two steps.

- The inverse nonlinear Fourier transform at the transmitter (INFT). At the transmitter, information is encoded in the nonlinear spectra of the signal according to a suitable constellation on $\left(\hat{X}(\lambda), \tilde{X}\left(\lambda_{j}\right)\right)$. The time domain signal is generated by taking the inverse nonlinear Fourier transform,

$$
q(t)=\operatorname{INFT}(\hat{X}(\lambda), \tilde{X}(\lambda)),
$$

and is sent over the channel. (The INFT is described formally in the next section.)

- The forward nonlinear Fourier transform at the receiver $(N F T)$. At the receiver, the (forward) nonlinear Fourier transform of the signal,

$$
(\hat{Y}(\lambda), \tilde{Y}(\lambda))=\operatorname{NFT}(q(t, z))
$$

is taken and the resulting spectra are compared against the transmitted spectra according to some metric $d(\hat{X}(\lambda), \tilde{X}(\lambda) ; \hat{Y}(\lambda), \tilde{Y}(\lambda))$.

As $q(t, 0)$ propagates in a communication network modeled by a complicated integrable equation, it is significantly distorted and undergoes inter-symbol interference (ISI) and interchannel interference. In the spectral domain, in the absence of noise, all the nonlinear spectral components propagate independent of each other and the channel is decomposed into a number of linear parallel independent channels. By diagonalizing the channel in this way, the deterministic ISI and inter-channel interference are removed in the spectral domain.

In this scheme, as in linear OFDM, communication objectives, such as constellation design, coding and modulation are entirely formulated in the spectral domain. All available degrees-of-freedom, i.e., $\left\{\lambda_{j}, \tilde{X}\left(\lambda_{j}\right), \hat{X}(\lambda)\right\}$ can be generally modulated. Time domain constraints can be translated to constraints in the spectral domain. A power constraint, for instance, can be exactly transformed to a power constraint in the spectral domain with the help of the Parseval's identity. A bandwidth limitation is not directly and simply expressed in the nonlinear spectral domain; however, it appears that the nonlinear spectrum of a signal bandlimited to $W$ is indeed confined, approximately, to a vertical strip in the real line and the upper half complex plane, with a width depending on $W$.

Fig. 5 illustrates the NFDM channel model that we use in this paper (and its subsequent parts) for data transmission over integrable channels such as optical fibers. Although in this paper we introduced the continuous-time NFT on the real line $t \in \mathbb{R}$, for practical implementation the nonlinear Fourier transform of discrete-time signals with periodic boundary conditions should be used; see [Part III] and references therein. 


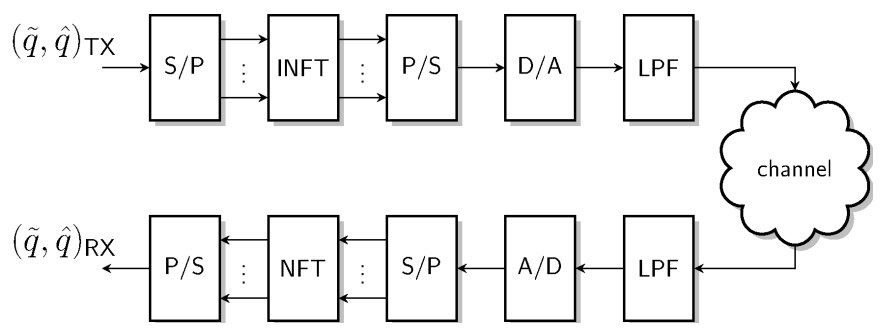

Fig. 5. Nonlinear frequency-division multiplexing system architecture.

\section{INVERSE NONLINEAR FOURIER TRANSFORM}

In this section, we describe the inverse nonlinear Fourier transform, which is a method for recovering the signal $q(t, \cdot)$ from its nonlinear Fourier transform $\left(\hat{q}(\lambda), \tilde{q}\left(\lambda_{j}\right)\right)$. This is the opposite of what was done in the Section [V] and it gives further important insight into how the NFT works. As in Section IV, the value of $z$ is irrelevant here and the index $z$ is thus suppressed.

\section{A. Riemann-Hilbert Factorization}

In the 1950s, before the publication of Gardner [16], a method for retrieving the potential $q(t)$ in the linear Schrödinger equation from the knowledge of the scattering matrix $S$ was already known in quantum mechanics. It was later realized that this method is an instance of a RiemannHilbert problem in complex analysis [9], [10]. Alternatively, the same task can be accomplished using the Gelfand-Levitan method [9], a different approach developed earlier in the context of inverse problems for Sturm-Liouville differential equations. Although either of these methods can be used for solving the inverse problem, in this paper we will use the Riemann-Hilbert method.

To begin, canonical eigenvectors can be found in two ways. On the one hand, they are related to $q(t)$ through the ZakharovShabat system (16). One can solve (16) to explicitly express the canonical eigenvectors as a series involving $q(t)$. On the other hand, canonical eigenvectors are related to the nonlinear Fourier transform via the projection equations (19a)-19b. The latter are two equations for four unknowns and in general cannot be solved. However, the unique properties of the canonical eigenvectors, which are analytic in disjoint regions of the complex plane, will allow us to find them in terms of the nonlinear Fourier transform. Note that this second derivation does not explicitly depend on $q(t)$; however, by equating the canonical eigenvectors obtained from these two derivations, we can relate the signal $q(t)$ to its nonlinear Fourier transform $\left(\hat{q}(\lambda), \tilde{q}\left(\lambda_{j}\right)\right)$.

We find it helpful to briefly introduce the tool that we employ in this section, namely the Riemann-Hilbert factorization problem in complex analysis.

Definition 5 (Riemann-Hilbert factorization). The RiemannHilbert factorization problem consists of finding two complex functions $f^{-}(z)$ and $f^{+}(z)$, respectively, analytic inside and outside of a closed contour $C$ in the complex plane, such that on $C$ they satisfy the boundary condition $f^{+}(z)=g(z) f^{-}(z)$ for some given $g(z)$.
It $g(z)$ satisfies a Hölder condition, it is possible to find both $f^{+}(z)$ and $f^{-}(z)$ everywhere in the complex plane from the given boundary condition. In Appendix C. we provide a brief overview of this problem and its solution. See [26] for more discussion.

\section{B. The Inverse Transform}

The inverse transform maps the two spectral functions $\left(\hat{q}(\lambda), \tilde{q}\left(\lambda_{j}\right)\right)$ to the signal $q(t)$.

As noted, we need to express the canonical eigenvectors in terms of the nonlinear Fourier transform. Below we will find it more convenient to work with canonical eigenvectors subject to fixed boundary conditions. Scaling the canonical eigenvectors as

$$
\begin{array}{ll}
V^{1}=v^{1} e^{-j \lambda t}, & \tilde{V}^{1}\left(t, \lambda^{*}\right)=\tilde{v}^{1}\left(t, \lambda^{*}\right) e^{j \lambda t}, \\
V^{2}=v^{2} e^{j \lambda t}, & \tilde{V}^{2}\left(t, \lambda^{*}\right)=\tilde{v}^{2}\left(t, \lambda^{*}\right) e^{-j \lambda t},
\end{array}
$$

the projection equations (19a) and 19b are transformed to

$$
\begin{aligned}
V^{2}(t, \lambda) & =a(\lambda) \tilde{V}^{1}\left(t, \lambda^{*}\right)+b(\lambda) e^{2 j \lambda t} V^{1}(t, \lambda), \\
\tilde{V}^{2}\left(t, \lambda^{*}\right) & =-b^{*}\left(\lambda^{*}\right) e^{-2 j \lambda t} \tilde{V}^{1}\left(t, \lambda^{*}\right)+a^{*}\left(\lambda^{*}\right) V^{1}(t, \lambda) .
\end{aligned}
$$

Lemma 5. If $q \in L^{1}(\mathbb{R})$, then $V^{1}(t, \lambda)$ and $V^{2}(t, \lambda)$ are analytic functions of $\lambda$ in the upper half complex plane $\mathbb{C}^{+}$ while $\tilde{V}^{1}\left(t, \lambda^{*}\right)$ and $\tilde{V}^{2}\left(t, \lambda^{*}\right)$ are analytic functions of $\lambda$ in the lower half complex plane.

Proof: See Appendix D

Rearranging 28a - 28b gives

$$
\begin{aligned}
\left(V^{1}(t, \lambda) V^{2}(t, \lambda)\right) & =\left(\begin{array}{cc}
\tilde{V}^{1}\left(t, \lambda^{*}\right) & \tilde{V}^{2}\left(t, \lambda^{*}\right)
\end{array}\right) \\
& \times\left(\begin{array}{cc}
\frac{b^{*}\left(\lambda^{*}\right)}{a^{*}\left(\lambda^{*}\right)} e^{-2 j \lambda t} & \frac{1}{a^{*}\left(\lambda^{*}\right)} \\
\frac{1}{a^{*}\left(\lambda^{*}\right)} & \frac{b(\lambda)}{a^{*}\left(\lambda^{*}\right)} e^{2 j \lambda t}
\end{array}\right) .
\end{aligned}
$$

Since $\left(V^{1}(t, \lambda) V^{2}(t, \lambda)\right)$ and $\left(\tilde{V}^{1}\left(t, \lambda^{*}\right) \tilde{V}^{2}\left(t, \lambda^{*}\right)\right)$ are analytic, respectively, in the upper and lower half complex plane, (29) defines a matrix Riemann-Hilbert problem.

Solution of the Riemann-Hilbert factorization problem 29. is given in Appendix $\mathrm{F}$ Omitting the details, the following linear system of equations, known as the Riemann-Hilbert system, is obtained:

$$
\begin{aligned}
\tilde{V}^{1}\left(t, \lambda^{*}\right)= & \left(\begin{array}{l}
1 \\
0
\end{array}\right)+\sum_{i=1}^{N} \frac{\tilde{q}\left(\lambda_{i}\right) e^{2 j \lambda_{i} t} V^{1}\left(t, \lambda_{i}\right)}{\lambda-\lambda_{i}} \\
& +\frac{1}{2 \pi j} \int_{-\infty}^{\infty} \frac{\hat{q}(\zeta) e^{2 j \zeta t} V^{1}(t, \zeta)}{\zeta-(\lambda-j \epsilon)} \mathrm{d} \zeta, \\
V^{1}(t, \lambda)= & \left(\begin{array}{l}
0 \\
1
\end{array}\right)-\sum_{i=1}^{N} \frac{\tilde{q}^{*}\left(\lambda_{i}^{*}\right) e^{-2 j \lambda_{i}^{*} t} \tilde{V}^{1}\left(t, \lambda_{i}^{*}\right)}{\lambda-\lambda_{i}^{*}} \\
& +\frac{1}{2 \pi j} \int_{-\infty}^{\infty} \frac{\hat{q}^{*}(\zeta) e^{-2 j \zeta t} \tilde{V}^{1}\left(t, \zeta^{*}\right)}{\zeta-(\lambda+j \epsilon)} \mathrm{d} \zeta, \\
\tilde{V}^{1}\left(t, \lambda_{m}^{*}\right)= & \left(\begin{array}{l}
1 \\
0
\end{array}\right)+\sum_{i=1}^{N} \frac{\tilde{q}\left(\lambda_{i}\right) e^{2 j \lambda_{i} t} V^{1}\left(t, \lambda_{i}\right)}{\lambda_{m}^{*}-\lambda_{i}}
\end{aligned}
$$




$$
\begin{aligned}
& +\frac{1}{2 \pi j} \int_{-\infty}^{\infty} \frac{\hat{q}(\zeta) e^{2 j \zeta t} V^{1}(t, \zeta)}{\zeta-\lambda_{m}^{*}} \mathrm{~d} \zeta, \\
V^{1}\left(t, \lambda_{m}\right)= & \left(\begin{array}{l}
0 \\
1
\end{array}\right)-\sum_{i=1}^{N} \frac{\tilde{q}^{*}\left(\lambda_{i}^{*}\right) e^{-2 j \lambda_{i}^{*} t} \tilde{V}^{1}\left(t, \lambda_{i}^{*}\right)}{\lambda_{m}-\lambda_{i}^{*}} \\
& +\frac{1}{2 \pi j} \int_{-\infty}^{\infty} \frac{\hat{q}^{*}(\zeta) e^{-2 j \zeta t} \tilde{V}^{1}(t, \zeta *)}{\zeta-\lambda_{m}} \mathrm{~d} \zeta,
\end{aligned}
$$

where $\lambda, \zeta \in \mathbb{R}, \lambda_{m} \in \mathbb{C}^{+}$and $m=1, \ldots, N$. This is a system of $2 N+2$ linear equations for $2 N+2$ discrete and continuous canonical eigenvectors $\left\{V^{1}\left(t, \lambda_{m}\right)\right\}_{m=1}^{N}$, $\left\{\tilde{V}^{1}\left(t, \lambda_{m}\right)\right\}_{m=1}^{N}$, and $V^{1}(t, \lambda)$ and $\tilde{V}^{1}(t, \lambda)$ as a function of the Fourier transform $\left(\hat{q}(\lambda), \tilde{q}\left(\lambda_{j}\right)\right)$.

Next, the canonical eigenvectors are related to the signal $q(t)$. By inspecting the Zakharov-Shabat system (16), it is shown in Appendix E that for $|\lambda| \gg 1$

$$
V^{1}(t, \lambda)=\left(\begin{array}{c}
\frac{1}{2 j \lambda} q(t) \\
1+\frac{1}{2 j \lambda} \int_{t}^{\infty}|q(t)|^{2} \mathrm{~d} t
\end{array}\right)+O\left(\lambda^{-2}\right) .
$$

It now becomes easy to recover the signal $q(t)$ from the nonlinear Fourier transform $\left(\hat{q}(\lambda), \tilde{q}\left(\lambda_{j}\right)\right)$. Eigenvector $V^{1}$ is related to $q$ via (31) for $|\lambda| \gg 1$, and is related to the nonlinear Fourier transform via (30b). Approximating $1 /(\lambda-\zeta)$ and $1 /\left(\lambda-\lambda_{i}\right)$ by $1 / \lambda+O\left(\lambda^{-2}\right)$ in (30b) for $|\lambda| \gg 1$ and comparing the resulting $V_{1}^{1}$ with $V_{1}^{1}$ in (31), we obtain

$$
\begin{aligned}
q^{*}(t)= & 2 j \sum_{i=1}^{N} \tilde{q}\left(\lambda_{i}\right) e^{2 j \lambda_{i} t} V_{2}^{1}\left(t, \lambda_{i}\right) \\
& -\frac{1}{\pi} \int_{-\infty}^{\infty} \hat{q}(\lambda) e^{2 j \lambda t} V_{2}^{1}(t, \lambda) \mathrm{d} \lambda .
\end{aligned}
$$

This represents $q(t)$ as a function of the nonlinear Fourier transform.

In summary, given $\left(\hat{q}(\lambda), \tilde{q}\left(\lambda_{j}\right)\right)$, we first solve the Riemann-Hilbert system to find the eigenvector $V^{1}$. For this purpose, one can discretize the system (30a)-30d) and solve a linear system of equations of the form

$$
A x=b,
$$

for appropriate $A$ and $b$. This is done for each fixed $t_{i}$ to determine values of $V^{l}\left(t_{i}, \lambda\right)$ at all times. Then, $V_{2}^{1}, \hat{q}$ and $\tilde{q}$ are substituted in (32) to obtain the signal $q(t)$.

Note that this inverse transform is taken only once at the transmitter to synthesize the desired pulse shapes. It is only the forward transform which needs to be taken in real time at the receiver.

Remark 1. In this paper we fixed $L$ to be the AKNS operator (14). In general, however $L$ needs to have adequate analyticity properties so that the inverse NFT can be carried out according to a Riemann-Hilbert factorization. Thus the existence of a Lax pair alone is not sufficient for the NFT.

The mathematical tools developed in this paper are used in Parts II and III of this paper to present further details pertaining the application of the suggested scheme in data transmission over integrable channels.

In [Part II] numerical methods are suggested to compute the nonlinear Fourier transform of a signal with respect to the AKNS Lax operator. We use the results of Parts I and II in [Part III] to study the NFT in the presence of the noise and illustrate the performance of the method in actual fiber-optic systems.

\section{CONCLUSIONS}

The nonlinear Fourier transform of a signal with respect to an operator $L$ in a Lax pair consists of continuous and discrete spectral functions $\hat{x}(\lambda)$ and $\tilde{x}\left(\lambda_{j}\right)$, obtainable by solving the eigenproblem for the $L$ operator. The NFT maps a Lax convolution to a multiplication operator in the spectral domain. Using the nonlinear Fourier transform, we propose a transmission scheme for integrable channels, termed nonlinear frequency-division multiplexing, in which the information in encoded in the nonlinear spectrum of the signal. The scheme is an extension of conventional OFDM to integrable channels generated by Lax pair operators. An example is the optical fiber channel, in which signals propagate according to the nonlinear Schrödinger equation. The class of integrable channels, though nonlinear and often complicated, are somehow "linear in disguise," and thus admit the proposed nonlinear frequencydivision multiplexing transmission scheme.

\section{APPENDIX A}

Spectrum of Bounded Linear Operators

When moving from finite-dimensional spaces (of e.g., matrices) to infinite-dimensional spaces (of $e . g$., functions and operators), some results do not carry over necessarily. Here we recall a few useful results in functional analysis [20].

Let $\mathcal{H}$ be a Hilbert space, let $\mathcal{D}$ be a dense subset of $\mathcal{H}$, and let $L: \mathcal{D} \rightarrow \mathcal{H}$ be an operator.

Definition 6. The adjoint of $L$ is the operator $L^{*}$ whose domain $\mathcal{D}^{*}$ consists of all $\psi \in \mathcal{H}$ for which there exists an element $L^{*} \psi \in \mathcal{H}$ such that

$$
\left\langle L^{*} \psi, \phi\right\rangle=\langle\psi, L \phi\rangle, \quad \forall \phi \in \mathcal{D} .
$$

The operator $L$ is said to be self-adjoint if $\mathcal{D}=\mathcal{D}^{*}$ and $L^{*}=L$. Ignoring domain subtleties (as is the case for bounded operators), self-adjoint operators are the analogue of Hermitian matrices.

Definition 7. Given an operator $L$ on a Hilbert space, an operator $M$ is said to be the inverse of $L$ if the domain of $M$ is the range of $L$, the range of $M$ is the domain of $L$, and $M L=I$ and $L M=I$.

We will restrict ourselves now to operators whose $\mathcal{D}$ is the entire space $\mathcal{H}$.

An operator $L: \mathcal{H} \rightarrow \mathcal{H}$ is invertible if it 1) is one-to-one 2) is onto 3) has bounded inverse. In finite-dimensional spaces, only the first condition is required.

An operator is bounded if it maps bounded inputs to bounded outputs. A bounded operator is invertible if it is oneto-one and onto. 
Definition 8. The spectrum of an operator $L$ on $\mathcal{H}$ is defined as

$$
\sigma(L)=\{\lambda \in \mathbb{C} \mid L-\lambda I \text { is not invertible }\} .
$$

The spectrum of a bounded operator can be partitioned into two classes, depending on the reason that $L-\lambda I$ fails to be invertible.

A complex number $\lambda$ is considered part of the discrete spectrum if $L-\lambda I$ is not one-to-one, i.e., $L \psi=\lambda \psi$ has a nonzero solution $\psi \in H$. In this case, $\lambda$ is called an eigenvalue, and each $\psi$ satisfying this equation for the given $\lambda$ is an associated eigenvector. The set of all eigenvalues is called the point spectrum or discrete spectrum of $L, \sigma_{\mathrm{disc}}(L)$.

It can also happen that $L-\lambda I$ fails to be surjective, i.e., the range of $L-\lambda I$ is a proper subset of $\mathcal{H}$. In this paper, we call the set of $\lambda$ for which this happens the continuous spectrum $\sigma_{\text {cont }}(L)$ [20]. Some authors subdivide what we refer to as the continuous spectrum into further classes (e.g., residual spectrum, essential spectrum, etc.) [20]; however, for the purposes of this paper, classification into discrete and continuous spectra will suffice.

In finite-dimensional Hilbert spaces the spectrum is entirely discrete. This may no longer be true in infinite-dimensional spaces, where the eigenvalues (if they exist) may only be one part of the spectrum. The spectrum of a self-adjoint operator is real.

The following examples illustrate some of these possibilities.

Example 5. The operator $L(x(t))=t x(t), x(t) \in L^{2}[0,1]$, has no eigenvalues and its spectrum is purely continuous $\sigma(L)=[0,1]$.

Example 6. The Fourier transform operator $\mathcal{F}(q)(\omega)=$ $\int_{-\infty}^{\infty} q(t) e^{j \omega t} \mathrm{~d} t$, regarded as on operator on $L^{2}(\mathbb{R})$ has the property $\mathcal{F}^{4}=I$. The eigenvalues are therefore the discrete values $\{ \pm 1, \pm j\}$. If $p(t)$ is an arbitrary polynomial, then $p(t) \exp \left(-t^{2} / 2\right)$ is an eigenfunction.

Example 7. Let $\nabla^{2}$ be the Laplace operator and let $r$ denote the radial distance in the three-dimensional space. The operator $L=-\nabla^{2}-\frac{1}{r}$ is self-adjoint, and therefore it has a real spectrum. The continuous spectrum is $[0, \infty)$ and the discrete spectrum is given by $\lambda_{n}=-\alpha \frac{1}{n^{2}}, n=1,2, \ldots$, for some constant $\alpha$.

Example 8. It is possible that the discrete spectrum of an operator is uncountable. For example, for a sequence $\left(x_{0}, x_{1}, \ldots\right) \in \ell^{2}=\left\{x: \sum_{i}\left|x_{i}\right|^{2}<\infty\right\}$, let the left-shift operator $L$ be defined as $L\left(x_{0}, x_{1}, x_{2}, \ldots\right)=\left(x_{1}, x_{2}, \ldots\right)$. The spectrum consists of the unit disk $|\lambda| \leqslant 1$. The portion $|\lambda|<1$ is the discrete spectrum while $|\lambda|=1$ is the continuous spectrum. The adjoint of $L$ is the right-shift operator $R\left(x_{0}, x_{1}, x_{2}, \ldots\right)=\left(0, x_{0}, x_{1}, \ldots\right)$. This operator has the same total spectrum, but it is entirely continuous.

Hermitian matrices are always diagonalizable, have real eigenvalues, and possess a complete set of orthonormal eigenvectors, which provide a basis for the input space. There is a perfect generalization of this result to compact self-adjoint operators [20].

Theorem 6 (Hilbert-Schmidt spectral theorem). Let $L$ be a compact self-adjoint operator in $\mathcal{H}$. Then it is always possible to find eigenvectors $\left\{\psi_{n}\right\}$ of $L$ forming an orthonormal basis for $\mathcal{H}$.

The spectral theory of operators that are not compact and self-adjoint is more involved; see [20].

An important class of operators are the multiplication operators, which are analogous to diagonal matrices.

Definition 9 (Multiplication operator). Let $f(t)$ be an arbitrary function. The operator $L$ defined by $\left(L_{f} \psi\right)(t)=f(t) \psi(t)$, which performs sample-wise multiplication, is called the $m u l$ tiplication operator or diagonal operator induced by $f(t)$.

Theorem 7. Every bounded self-adjoint operator in a separable Hilbert space $\mathcal{H}$ is unitarily equivalent to a multiplication operator $\Gamma$, i.e., $L=U \Gamma U^{-1}$ where $U$ is unitary.

It follows that the essence of a bounded self-adjoint operator is just a multiplication operator.

\section{APPENDIX B}

\section{Proof of ElEMENTARY PROPERTIES OF THE NFT}

In this section, we sketch the proofs of the properties of the NFT stated in Section IV-D.

1) If $\|q(t)\|_{L^{1}} \ll 1$, then $y^{2}(t, \lambda)$ and $q^{2}(t)$ terms can be ignored in (22) and (23). From the resulting equations, it follows that there is no discrete spectrum and $\hat{q}(\lambda) \rightarrow$ $Q(\lambda)$. The quadratic terms are introduced by the NFT to account for the nonlinearity.

2) This follows from 1) in above, and that when $|a| \ll 1$, the squared terms representing the nonlinearity can be ignored.

3) This follows from replacing $q(t)$ with $e^{j \phi} q(t)$ in 22 and (23). Alternatively, it can be proved by changing variables $u=\operatorname{diag}(1, \exp (j \phi)) v$ in $v_{t}=P\left(\lambda, q e^{j \phi}\right) v$ to get $u_{t}=P(\lambda, q) u$ with the same boundary condition (17b). Since $u_{1}=v_{1}$ and $u_{2}=\exp (j \phi) v_{2}, a(\lambda)$ does not change and $b(\lambda)$ is scaled by $e^{-j \phi}$.

4) This follows from noting that a) the eigenproblem 16 is invariant under transformation $t^{\prime}=t / a, q^{\prime}=a q$ and $\lambda^{\prime}=a \lambda$, and $\mathrm{b}$ ) all boundary conditions are invariant under this transformation since $\exp \left( \pm j \lambda^{\prime} t^{\prime}\right)=\exp ( \pm j \lambda t)$. Note that if $\operatorname{sgn}(a)<0$, boundary conditions at $\pm \infty$ are interchanged.

$5,6)$ Property 5) and 6) follow by replacing $q(t)$ with $q\left(t-t_{0}\right)$ and $e^{-2 j \omega t} q(t)$ in (22) and (23), and accordingly changing variables. Alternatively, Property 5) follows by noting the structure of the propagator in Property 1 below. Property 6) can also be proved by noting that if $v_{t}=$ $P\left(\lambda, q e^{-2 j \omega t}\right) v$ and $v(t \rightarrow \infty, \lambda) \rightarrow(1,0)^{T} \exp (-j \lambda t)$, then $u=\operatorname{diag}(\exp (j \omega t), \exp (-j \omega t)) v$ satisfies $u_{t}=$ $P\left(\lambda^{\prime}, q\right) u$ and $u(t \rightarrow \infty, \lambda) \rightarrow(1,0)^{T} \exp \left(-j \lambda^{\prime} t\right)$, for $\lambda^{\prime}=\lambda-\omega$.

7) This is the statement of (26). 
8) The following identity, known as the trace formula, can be easily proved for the nonlinear Fourier transform [8]

$$
c_{n}=\frac{4}{n} \sum_{i=1}^{N} \Im\left(\lambda_{i}^{n}\right)+\frac{1}{\pi} \int_{-\infty}^{\infty} \lambda^{n-1} \log \left(1+|\hat{q}(\lambda)|^{2}\right) \mathrm{d} \lambda .
$$

Here $c_{n}$ are the secondary constants of motion, i.e., quantities, directly in terms of the time domain data, which are preserved during the flow of the NLS equation. The first few ones are the energy

$$
c_{1}=\int_{-\infty}^{\infty}|q(t)|^{2} \mathrm{~d} t
$$

momentum

$$
c_{2}=\frac{1}{2 j} \int_{-\infty}^{\infty} q(t) q_{t}^{*}(t) \mathrm{d} t
$$

and the Hamiltonian

$$
c_{3}=-\frac{1}{4} \int_{-\infty}^{\infty}\left(|q(t)|^{4}-\left|q_{t}(t)\right|^{2}\right) \mathrm{d} t .
$$

Parseval's identity is the trace formula at $n=1$.

The other two properties related to NFT can be proved too.

1) Let $q(t)$ be supported in the interval $\left[t_{1}, t_{2}\right]$ and let $K\left(q(t), t_{1}, t_{2}\right)$ denote a propagator (linear transformation) which maps $v(t, \lambda)$ in (16) from $t=t_{1}$ to $t=t_{2}$, i.e., $v\left(t_{2}, \lambda\right)=K v\left(t_{1}, \lambda\right)$. The propagator is structured as

$$
K=\left(\begin{array}{cc}
a(\lambda) e^{-j \lambda\left(t_{2}-t_{1}\right)} & -b^{*}\left(\lambda^{*}\right) e^{-j \lambda\left(t_{2}+t_{1}\right)} \\
b(\lambda) e^{j \lambda\left(t_{2}+t_{1}\right)} & a^{*}\left(\lambda^{*}\right) e^{j \lambda\left(t_{2}-t_{1}\right)}
\end{array}\right) .
$$

Let $q_{1}(t)$ and $q_{2}(t)$, supported, respectively, in the intervals $\left[t_{1}, t_{2}\right]$ and $\left[t_{2}, t_{3}\right], t_{1}<t_{2}<t_{3}$, correspond to the propagators $K_{1}\left(q_{1}(t), t_{1}, t_{2}\right)$ and $K_{2}\left(q_{2}(t), t_{2}, t_{3}\right)$. Then $q(t)=q_{1}(t)+q_{2}(t)$ is supported in $\left[t_{1}, t_{3}\right]$, and from linearity corresponds to the propagator $K=K_{2} K_{1}$

$$
\begin{aligned}
K= & \left(\begin{array}{cc}
a_{2}(\lambda) e^{-j \lambda\left(t_{3}-t_{2}\right)} & -b_{2}^{*}\left(\lambda^{*}\right) e^{-j \lambda\left(t_{3}+t_{2}\right)} \\
b_{2}(\lambda) e^{j \lambda\left(t_{3}+t_{2}\right)} & a_{2}^{*}\left(\lambda^{*}\right) e^{j \lambda\left(t_{3}-t_{2}\right)}
\end{array}\right) \\
& \times\left(\begin{array}{cc}
a_{1}(\lambda) e^{-j \lambda\left(t_{2}-t_{1}\right)} & -b_{1}^{*}\left(\lambda^{*}\right) e^{-j \lambda\left(t_{2}+t_{1}\right)} \\
b_{1}(\lambda) e^{j \lambda\left(t_{2}+t_{1}\right)} & a_{1}^{*}\left(\lambda^{*}\right) e^{j \lambda\left(t_{2}-t_{1}\right)}
\end{array}\right) .
\end{aligned}
$$

The $1 \times 1$ and $2 \times 1$ elements are

$$
\begin{aligned}
& K_{11}=e^{-j \lambda\left(t_{3}-t_{1}\right)}\left(a_{1}(\lambda) a_{2}(\lambda)-b_{1}(\lambda) b_{2}^{*}\left(\lambda^{*}\right)\right), \\
& K_{21}=e^{j \lambda\left(t_{3}+t_{1}\right)}\left(a_{1}(\lambda) b_{2}(\lambda)+b_{1}(\lambda) a_{2}^{*}\left(\lambda^{*}\right)\right) .
\end{aligned}
$$

Comparing with $K_{11}=a(\lambda) e^{-j \lambda\left(t_{3}-t_{1}\right)}$ and $K_{21}=$ $b(\lambda) e^{j \lambda\left(t_{3}+t_{1}\right)}$, we get the desired result.

2) See [25].

\section{APPENDIX C \\ RiEMANN-Hilbert FACTORIZATION PROBLEM}

Recall that a complex function $f(z)=u(x, y)+j v(x, y)$ in the complex plane $z=(x, y)$ is differentiable at a point $\left(x_{0}, y_{0}\right)$ if and only if the partial derivatives $u_{x}, u_{y}, v_{x}$ and $v_{y}$ are continuous and

$$
u_{x}=v_{y}, \quad u_{y}=-v_{x} .
$$

The compatibility conditions (33) are called the CauchyRiemann conditions and are obtained by equating the limit $\Delta z \rightarrow 0$ in the definition of derivative along the real and imaginary axes. This means that, unlike real functions, differentiability of a complex function imposes a constraint between the real and imaginary parts of the function.

A function $f(z)$ is said to be analytic at $\left(x_{0}, y_{0}\right)$ if it is differentiable in a neighborhood of that point. If $f(z)$ is analytic in an open region $\Omega$ of the complex plane, then a power series representation of $f(z)$ is convergent in $\Omega$. Existence of a power series representation has a number of interesting consequences. For instance, the set $S$ of the zeros of a function analytic in a nonempty connected open subset $\Omega$ of $\mathbb{C}$ is either the entire $\Omega$ or has no limit point in $\Omega$ (i.e., $S$ consists of countable isolated points in $\Omega$ ) [24].

\section{A. The Scalar Riemann-Hilbert Problem}

In the scalar Riemann-Hilbert (RH) problem, the task is to find functions $f^{+}(z)$ and $f^{-}(z)$, analytic, respectively, inside and outside of a given smooth closed contour $C$, such that on C

$$
f^{+}(t)=g(t) f^{-}(t)+h(t), \quad t \in C,
$$

where $h(t)$ and $g(t)$ (with $g(t) \neq 0$ for all $t \in C$ ), are given functions satisfying a Hölder condition on $C$.

A solution of this problem can be found in [26], [27]. For a brief review, in this section we follow [27].

The following lemma is central in solving the simplest scalar RH factorization.

Lemma 8 (Sokhotski-Plemelj formulae). Let $C$ be any smooth, closed, counter-clockwise, contour in the complex plane and let $f(x)$ be any function satisfying a Hölder condition on $C$ defined by

$$
|f(t)-f(\tau)| \leqslant k|t-\tau|^{\alpha}, k>0, \forall t, \tau \in C,
$$

for some $0<\alpha \leqslant 1$. Then the projection integral

$$
F(\zeta)=\frac{1}{2 \pi j} \oint_{C} \frac{f(z)}{z-\zeta} \mathrm{d} z
$$

is analytic everywhere in $\mathbb{C}$ except possibly at points $\zeta$ on the contour $C$ (where $F(\zeta)$ is not defined). If $\zeta$ approaches $C$ along a path entirely inside the contour $C$, then

$$
F^{+}(\zeta) \triangleq \lim _{z \rightarrow \zeta} F(\zeta)=\frac{f(\zeta)}{2}+\frac{1}{2 \pi j} \text { p.v. } \int_{C} \frac{f(z)}{z-\zeta} \mathrm{d} z .
$$


If $\zeta$ approaches $C$ along a path entirely outside the contour $C$, then

$$
F^{-}(\zeta) \triangleq \lim _{z \rightarrow \zeta} F(\zeta)=-\frac{f(\zeta)}{2}+\frac{1}{2 \pi j} \text { p.v. } \int_{C} \frac{f(z)}{z-\zeta} \mathrm{d} z .
$$

Here p.v. denotes the principal value integral defined by

$$
\text { p.v. } \int_{C} \frac{f(z)}{z-\zeta} \mathrm{d} z=\lim _{\epsilon \rightarrow 0} \oint_{C-C_{\epsilon}} \frac{f(z)}{z-\zeta} \mathrm{d} z,
$$

in which $C_{\epsilon}$ is an infinitesimal part of $C$ centered at $z=\zeta$ and with length $2 \epsilon$.

Proof: See [26], [27].

The projected function $F(\zeta)$ is a sectionally analytic function of $\zeta$ with respect to $C$, i.e., it is analytic in sections $C^{+}$ (the interior of $C$ ) and $C^{-}$(the exterior of $C$ ), and the limits $F^{ \pm}(\zeta)$ exist (as given by (36) and (37)).

A consequence of Lemma 8 is that $F^{ \pm}(\zeta)$ satisfy the following jump condition on the boundary $C$

$$
F^{+}(t)-F^{-}(t)=f(t) .
$$

The projection operator therefore produces functions which are analytic almost everywhere, except on a contour where it experiences a jump in its limits.

Both unknowns $f^{+}(z)$ and $f^{-}(z)$ in the scalar RH problem can be determined from the single boundary equation (34), using the projection operator (35). To see this, first consider the homogeneous case where $h(t)=0$. One can rewrite 34 as a jump condition

$$
\log f^{+}(z)-\log f^{-}(z)=\log g(z), \quad z \in C .
$$

Functions $\log f^{+}(z)$ and $\log f^{-}(z)$ can be viewed as portions of a single sectionally analytic function $\log f(z)$ which is analytic in $C^{+}$and $C^{-}$and on boundary $C$ its limits jump as $\log g(t)$. In view of the projection operator $P(\underline{35}$ ), consider

$$
\log f(z)=\frac{1}{2 \pi j} \oint_{C} \frac{\log g(\lambda)}{\lambda-z} \mathrm{~d} \lambda .
$$

If $\log g(t)$ satisfies a Hölder condition on $C$, then $\log f(z)$ is analytic strictly inside and outside $C$. On $C$, we can define $\log f^{+}(z)$ and $\log f^{-}(z)$, respectively, as equal to the limits (36) and (37). The function obtained in this way satisfies (34) and has the desired analyticity properties.

Note however that, unlike $g(t), \log g(t)$ in the integrand may not satisfy a Hölder condition. To resolve this issue, we can multiply $g(t)$ by a decaying factor $t^{-k}$, for a suitable $k$, to make $t^{-k} g(t)$ Hölder, and obtain $f^{+}(t)=$ $\left(t^{-k} g(t)\right)\left(t^{k} f^{-}(z)\right)$. Therefore, defining

$$
F(z)=\exp \left(\frac{1}{2 \pi j} \oint_{C} \frac{\log \lambda^{-k} g(\lambda)}{\lambda-z} \mathrm{~d} \lambda\right),
$$

we have the following solution for the homogeneous RH problem:

$$
f^{+}(z)= \begin{cases}F(z), & z \in C^{+} \\ F^{+}(z), & z \in C\end{cases}
$$

and

$$
f^{-}(z)= \begin{cases}z^{-k} F(z), & z \in C^{-}, \\ z^{-k} F^{-}(z), & z \in C .\end{cases}
$$

Here $k$ can be chosen so that $t^{-k} g(t)$ is continuous and the total phase change of $\log t^{-k} g(t)$ is zero along the closed path $C$.

The solution $f^{ \pm}(z)$ is called the fundamental solution to the scalar RH problem. From the homogeneity of (34), one can obtain other solutions by multiplying $f^{ \pm}(z)$ by any entire function in $C$.

We can generalize the above procedure to solve the nonhomogeneous Riemann-Hilbert problem (34). In this case, we can find a factorization $g(t)=g^{+}(t) / g^{-}(t)$ by solving a homogeneous Riemann-Hilbert problem with boundary conditions $g^{+}(t)=g(t) g^{-}(t)$. Then (34) is reduced to

$$
\frac{f^{+}(t)}{g^{+}(t)}-\frac{f^{-}(t)}{g^{-}(t)}=\frac{h(t)}{g^{+}(t)}
$$

which, as before, can be solved in closed form using the Plemelj formulae.

\section{B. The Matrix Riemann-Hilbert Problem}

When formulating the inverse nonlinear Fourier transform, we face a matrix Riemann-Hilbert problem (29). Matrix RH problems are generally more involved and may not allow closed-form solutions [26]. As we will see in the Appendix E, for the particular matrix $\mathrm{RH}$ problem (29), the projection operator (35) is sufficient to solve the problem.

\section{APPENDIX D}

\section{PROOF OF LEMMA 5}

Analyticity of the canonical eigenvectors is directly a property of the Zakharov-Shabat system (16). A formal proof can be found in [8], [28]; we simplify and outline it in this section for the sake of completeness.

Consider the scaled canonical eigenvectors (27). Transforming the Zakharov-Shabat system (16), the scaled eigenvectors satisfy

$$
\begin{aligned}
& V_{t}^{2}=\left(\begin{array}{cc}
0 & q \\
-q^{*} & 2 j \lambda
\end{array}\right) V^{2}, \quad V^{2}(-\infty)=\left(\begin{array}{l}
1 \\
0
\end{array}\right), \\
& \tilde{V}_{t}^{2}=\left(\begin{array}{cc}
-2 j \lambda & q \\
-q^{*} & 0
\end{array}\right) \tilde{V}^{2}, \quad \tilde{V}^{2}(-\infty)=\left(\begin{array}{l}
0 \\
1
\end{array}\right) \text {, } \\
& \tilde{V}_{t}^{1}=\left(\begin{array}{cc}
0 & q \\
-q^{*} & 2 j \lambda
\end{array}\right) \tilde{V}^{1}, \quad \tilde{V}^{1}(\infty)=\left(\begin{array}{l}
1 \\
0
\end{array}\right) \text {, } \\
& V_{t}^{1}=\left(\begin{array}{cc}
-2 j \lambda & q \\
-q^{*} & 0
\end{array}\right) V^{1}, \quad V^{1}(\infty)=\left(\begin{array}{l}
0 \\
1
\end{array}\right) .
\end{aligned}
$$

Let us, for instance, solve for the canonical eigenvector $V^{2}$ in (38). Considering the $q$ terms as an external force and using the Duhamel's formula [29], (38) can be transformed into its integral representation

$$
\begin{aligned}
& V^{2}(t, \lambda)=\left(\begin{array}{l}
1 \\
0
\end{array}\right) \\
& \quad+\int_{-\infty}^{\infty} h\left(t-t^{\prime}, \lambda\right)\left(\begin{array}{cc}
0 & q\left(t^{\prime}\right) \\
-q^{*}\left(t^{\prime}\right) & 0
\end{array}\right) V^{2}\left(t^{\prime}, \lambda\right) \mathrm{d} t^{\prime},
\end{aligned}
$$


where the system "impulse response" $h(t, \lambda)$ is

$$
h(t, \lambda)=\left(\begin{array}{cc}
u(t) & 0 \\
0 & e^{2 j \lambda t} u(t)
\end{array}\right),
$$

where $u(t)$ is the step function and we have ignored transient terms since the boundary condition starts at $t=-\infty$.

The analyticity of eigenvectors can be seen intuitively at at this stage. The impulse response (40) involves the term $e^{2 j \lambda t} u(t)$ and hence $(38)$ is well defined in $\mathbb{C}^{+}$if $q \in L^{1}(\mathbb{R})$. The impulse response for the $V^{1}$ equation, by converting it to the $V^{2}$ equation, involves $-e^{-2 j \lambda t} u(-t)$ and hence it is bounded in the same region. The impulse response for $\tilde{V}^{2}$ and $\tilde{V}^{1}$ have terms proportional to $e^{-2 j \lambda t} u(t)$ and $-e^{2 j \lambda t} u(-t)$, respectively, and therefore these eigenvectors are analytic in $\mathbb{C}^{-}$for $q \in L^{1}(\mathbb{R})$.

A more precise argument proceeds by solving (39) explicitly. Duhamel's integral (39) is of the form of a fixed-point map

$$
V^{2}=\left(\begin{array}{l}
1 \\
0
\end{array}\right)+T\left(V^{2}\right),
$$

where $T$ is the linear operator underlying the integral term in (39). A candidate for the solution is the sum

$$
V^{2}=\sum_{k=0}^{\infty} U^{k}
$$

where $U^{k}$ satisfy the iteration

$$
U^{k+1}=\left(\begin{array}{l}
1 \\
0
\end{array}\right)+T\left(U^{k}\right), \quad U_{0}=\left(\begin{array}{l}
0 \\
0
\end{array}\right) .
$$

The first few terms are

$$
\begin{aligned}
& U^{1}=\left(\begin{array}{l}
1 \\
0
\end{array}\right), \quad U^{2}=\left(\begin{array}{c}
0 \\
-\int_{t_{1}=-\infty}^{t} q^{*}\left(t_{1}\right) e^{2 j \lambda\left(t-t_{1}\right)} \mathrm{d} t_{1}
\end{array}\right), \\
& U^{3}=\left(\begin{array}{c}
-\int_{t_{2}=-\infty}^{t} \int_{t_{1}=-\infty}^{t_{2}} q\left(t_{2}\right) q^{*}\left(t_{1}\right) e^{2 j \lambda\left(t_{2}-t_{1}\right)} \mathrm{d} t_{1} \mathrm{~d} t_{2} \\
0
\end{array}\right),
\end{aligned}
$$

and the $k^{\text {th }}$ term is recursively defined by

$$
U^{k+1}=\left(\begin{array}{l}
1 \\
0
\end{array}\right)+\int_{-\infty}^{t}\left(\begin{array}{c}
q\left(t^{\prime}\right) U_{2}^{k}\left(t^{\prime}, \lambda\right) \\
-q^{*}\left(t^{\prime}\right) U_{1}^{k}\left(t^{\prime}, z\right) e^{2 j \lambda\left(t-t^{\prime}\right)}
\end{array}\right) \mathrm{d} t^{\prime} .
$$

By induction on $k$, as (44) suggests, if $U^{k}$ is analytic and $q(t) \in L^{1}(\mathbb{R})$, then $U^{k+1}$ is analytic. Since, the series (41) is uniformly convergent on $t, V^{2}$ is analytic in $\mathbb{C}^{+}$.

Similarly one proves the analyticity of the other canonical eigenvectors in their corresponding region.

\section{APPENDIX E}

ASYMPTOTIC FoRM OF CANONICAL EIGENVECTORS AND NONLINEAR FOURIER COEFFICIENTS WHEN $|\lambda| \gg 1$

If $\lambda \in C^{+}$and $|\lambda| \gg 1$, then $\frac{1}{j(\omega-2 \lambda)}=\frac{-1}{2 j \lambda}+O\left(\lambda^{-2}\right)$ and, taking the inverse Fourier transform, we can approximate $e^{-2 j \lambda\left(t_{1}-t\right)} u\left(t-t_{1}\right)=-\frac{1}{2 j \lambda} \delta\left(t_{1}-t\right)+O\left(\lambda^{-2}\right)$. Substituting into (41), (42), (43), for $|\lambda| \gg 1$ we obtain

$$
V^{2}(t, \lambda)=\left(\begin{array}{c}
1+\frac{1}{2 j \lambda} \int_{-\infty}^{t}|q(t)|^{2} \mathrm{~d} t \\
\frac{1}{2 j \lambda} q^{*}(t)
\end{array}\right)+O\left(\lambda^{-2}\right) .
$$

A similar asymptotic expression can be derived for $V^{1}(\lambda \gg 1)$

$$
V^{1}(t, \lambda)=\left(\begin{array}{c}
\frac{1}{2 j \lambda} q(t) \\
1+\frac{1}{2 j \lambda} \int_{t}^{\infty}|q(t)|^{2} \mathrm{~d} t
\end{array}\right)+O\left(\lambda^{-2}\right) .
$$

For the nonlinear Fourier coefficients, if $|\lambda| \rightarrow \infty, q$ can be assumed zero in (16) compared to $j \lambda$. Thus $v(t, \lambda)$ approaches the boundary conditions at $t= \pm \infty$. Therefore

$$
\begin{aligned}
a(\lambda) & =\left\langle v^{2}(t, \lambda), v^{1}(t, \lambda)\right\rangle_{s} \\
& =\left\langle v^{2}(+\infty, \lambda), v^{1}(+\infty, \lambda)\right\rangle_{s} \\
& \rightarrow\left\langle v^{2}(-\infty, \lambda), v^{1}(+\infty, \lambda)\right\rangle_{s}=1 .
\end{aligned}
$$

Similarly, it is shown that $b(\lambda) \rightarrow 0$ as $|\lambda| \rightarrow \infty$.

\section{APPENDIX F}

\section{SOLUTION OF THE RIEMANN-HILBERT PROBLEM}

In Section VII the inverse nonlinear Fourier transform was formulated as an instance of the Riemann-Hilbert factorization problem. Following the discussion in the Appendix C, the resulting factorization problem can be solved in a simplified manner via an appropriate contour integration.

Dividing both sides of the projection equations $119 \mathrm{a}-(19 \mathrm{~b})$ by $a(\lambda)(\lambda-\zeta)$, for parameter $\zeta \in C^{-}$, and integrating on the real axis $-\infty<\lambda<\infty$, we obtain

$$
\begin{gathered}
\frac{1}{2 \pi j} \int_{\lambda=-\infty}^{\infty} \frac{V^{2}(t, \lambda)}{a(\lambda)(\lambda-\zeta)} \mathrm{d} \lambda=\frac{1}{2 \pi j} \int_{\lambda=-\infty}^{\infty} \frac{\tilde{V}^{1}\left(t, \lambda^{*}\right)}{\lambda-\zeta} \mathrm{d} \lambda \\
+\frac{1}{2 \pi j} \int_{\lambda=-\infty}^{\infty} \frac{\hat{q}(\lambda) e^{2 j \lambda t} V^{1}(t, \lambda)}{\lambda-\zeta} \mathrm{d} \lambda,
\end{gathered}
$$

in which the integration is performed on the open path $z=\lambda$, $-\infty<\lambda<\infty$. The integration path thus passes the singularity $\lambda=\zeta$ from above in all the integrals.

Cauchy integrals in (47) are computed from the residue theorem. The integration path $-\infty<\lambda<\infty$ can be closed in the upper or lower half-planes. To compute the first integral, we close the path in the upper half-plane and denote the resulting closed contour by $\mathrm{C}_{-\zeta}^{+}$, i.e., the upper half plane 
and excluding the singularity $z=\zeta$

$$
\begin{aligned}
& \frac{1}{2 \pi j} \int_{-\infty}^{\infty} \frac{V^{2}(t, \lambda)}{a(\lambda)(\lambda-\zeta)} \mathrm{d} \lambda \\
= & \frac{1}{2 \pi j} \oint_{C_{-\zeta}^{+}} \frac{V^{2}(t, z)}{a(z)(z-\zeta)} \mathrm{d} z-\lim _{R \rightarrow \infty} \frac{1}{2 \pi j} \int_{R e^{j 0}}^{R e^{j \pi}} \frac{\left(\begin{array}{l}
1 \\
0
\end{array}\right)}{a(z)(z-\zeta)} \mathrm{d} z \\
= & \sum_{i=1}^{N} \frac{V^{2}\left(t, \lambda_{j}\right)}{a_{\lambda}\left(\lambda_{j}\right)\left(\lambda_{j}-\zeta\right)}-\frac{1}{2}\left(\begin{array}{l}
1 \\
0
\end{array}\right) \\
= & \sum_{i=1}^{N} \frac{b\left(\lambda_{i}\right) e^{2 j \lambda_{i} t} V^{1}\left(t, \lambda_{i}\right)}{a_{\lambda}\left(\lambda_{i}\right)\left(\lambda_{i}-\zeta\right)}-\frac{1}{2}\left(\begin{array}{l}
1 \\
0
\end{array}\right) \\
= & \sum_{i=1}^{N} \frac{\tilde{q}\left(\lambda_{i}\right) e^{2 j \lambda_{i} t} V^{1}\left(t, \lambda_{i}\right)}{\lambda_{i}-\zeta}-\frac{1}{2}\left(\begin{array}{l}
1 \\
0
\end{array}\right),
\end{aligned}
$$

where, in the second line, when $R \rightarrow \infty$, we have used the asymptotic values (45) and (46) in Appendix E. Note that we assumed that eigenvalues $\lambda_{j}$ are all simple zeros of $a(\lambda)$, i.e., no multiplicity.

To compute the second integral in (47), we close the integration path in the lower half-plane and denote the resulting closed contour by $C_{+\zeta}^{-}$, i.e., the lower half-plane and including the singularity $z=\zeta$

$$
\begin{aligned}
& \frac{1}{2 \pi j} \int_{\lambda=-\infty}^{\infty} \frac{\tilde{V}^{1}\left(t, \lambda^{*}\right)}{\lambda-\zeta} \mathrm{d} \lambda=\frac{1}{2 \pi j} \oint_{C_{+\zeta}^{-}} \frac{\tilde{V}^{1}\left(t, z^{*}\right)}{z-\zeta} \mathrm{d} z \\
& -\lim _{R \rightarrow \infty} \frac{1}{2 \pi j} \int_{R e^{j 2 \pi}}^{R e^{j \pi}} \frac{\left(\begin{array}{l}
1 \\
0
\end{array}\right)}{z-\zeta} \mathrm{d} z=-\tilde{V}^{1}\left(t, \zeta^{*}\right)+\frac{1}{2}\left(\begin{array}{l}
1 \\
0
\end{array}\right) .
\end{aligned}
$$

The last integral in 47 is not computed, because the boundedness of $e^{2 j \lambda t}$ depends on the sign of $t$. For $t>0$, we can consider $C_{-\zeta}^{+}$which leads to the expression (48) multiplied by $u(t)$. For $t<0$, we should inevitably consider $C_{+\zeta}^{-}$, where poles of $V^{1}(x, \lambda)$ are unknown. As a result, this integral is left untreated.

Using (48) and (49) in (47), we obtain an integral equation relating canonical eigenvectors $V^{1}$ and $\tilde{V}^{1}$ to $\hat{q}(\lambda)$ and $\tilde{q}\left(\lambda_{j}\right)$

$$
\begin{aligned}
\tilde{V}^{1}\left(t, \zeta^{*}\right)= & \left(\begin{array}{l}
1 \\
0
\end{array}\right)+\sum_{i=1}^{N} \frac{\tilde{q}\left(\lambda_{i}\right) e^{2 j \lambda_{i} t} V^{1}\left(t, \lambda_{i}\right)}{\zeta-\lambda_{i}} \\
& +\frac{1}{2 \pi j} \int_{\lambda=-\infty}^{\infty} \frac{\hat{q}(\lambda) e^{2 j \lambda t} V^{1}(t, \lambda)}{\lambda-(\zeta-j \epsilon)} \mathrm{d} \lambda .
\end{aligned}
$$

This is equation 30a in the Riemann-Hilbert system when $\zeta$ approaches the real line from below. Since (50) holds for any $\zeta \in C^{-}$, evaluating (50) at $\zeta=\lambda_{j}^{*}, j=1, \ldots, N$, produces (30c). The remaining equations are obtained by taking the tilde " $\sim$ " operation from these two equations and subsequently replacing $\lambda$ and $\lambda_{m}$ with, respectively, $\lambda^{*}$ and $\lambda_{m}^{*}$.

\section{REFERENCES}

[1] V. E. Zakharov and A. B. Shabat, "Exact theory of two-dimensional self-focusing and one-dimensional self-modulation of waves in nonlinear media," Soviet J. Exp. Theory Phys. (JETP), vol. 34, no. 1, pp. 62-69, Jan. 1972.

[2] G. P. Agrawal, Nonlinear Fiber Optics, 5th ed. San Francisco, CA, USA: Academic Press, 2012.

[3] R.-J. Essiambre, G. Kramer, P. J. Winzer, G. J. Foschini, and B. Goebel, "Capacity limits of optical fiber networks," IEEE J. Lightw. Technol., vol. 28, no. 4, pp. 662-701, Feb. 2010.

[4] G. Kramer, A. Ashikhmin, A. J. van Wijngaarden, and X. Wei, "Spectral efficiency of coded phase-shift keying for fiber-optic communication," IEEE J. Lightw. Technol., vol. 21, no. 10, pp. 2438-2445, Oct. 2003.

[5] E. Ip and J. M. Kahn, "Compensation of dispersion and nonlinear impairments using digital backpropagation," IEEE J. Lightw. Technol., vol. 26, no. 20, pp. 3416-3425, Oct. 2008.

[6] K. Roberts, C. Li, L. Strawczynski, M. O'Sullivan, and I. Hardcastle, "Electronic precompensation of optical nonlinearity," IEEE Photon. Technol. Lett., vol. 18, no. 2, pp. 403-405, Jan. 2006.

[7] L. Beygi, E. Agrell, M. Karlsson, and P. Johannisson, "Signal statistics in fiber-optical channels with polarization multiplexing and self-phase modulation," IEEE J. Lightw. Technol., vol. 29, no. 16, pp. 2379-2386, Aug. 2011.

[8] M. J. Ablowitz and H. Segur, Solitons and the Inverse Scattering Transform, ser. SIAM Stud. Appl. Numer. Math. Philadelphia, PA, USA: SIAM, 1981, vol. 4.

[9] L. D. Faddeev and L. A. Takhtajan, Hamiltonian Methods in the Theory of Solitons. Berlin, Germany: Springer-Verlag, 2007.

[10] T. Tao and C. Thiele, "Nonlinear Fourier analysis," Arxiv e-prints, arXiv:1201.5129, Jan. 2012. [Online]. Available: http://arxiv.org/abs/1201.5129

[11] M. I. Yousefi and F. R. Kschischang, "Information transmission using the nonlinear Fourier transform, Part II: Numerical methods," Arxiv e-prints, arXiv:1204.0830, Apr. 2012. [Online]. Available: http://arxiv.org/abs/1204.0830

[12] - "Information transmission using the nonlinear Fourier transform, Part III: Spectrum modulation," Arxiv e-prints, arXiv:1302.2875, Feb. 2013. [Online]. Available: http://arxiv.org/abs/1302.2875

[13] A. Hasegawa and T. Nyu, "Eigenvalue communication," IEEE J. Lightw. Technol., vol. 11, no. 3, pp. 395-399, Mar. 1993.

[14] E. Fermi, J. Pasta, and S. Ulam, "Studies of nonlinear problems," Los Alamos Scientific Laboratory Report No. LA-1940, Tech. Rep., May 1955.

[15] N. J. Zabusky and M. D. Kruskal, "Interaction of 'solitons' in a collisionless plasma and the recurrence of initial states," Phys. Rev. Lett., vol. 15, no. 6, pp. 240-243, Aug. 1965.

[16] C. S. Gardner, J. M. Greene, M. D. Kruskal, and R. M. Miura, "Method for solving the Korteweg-de Vries equation," Phys. Rev. Lett., vol. 19, no. 19, pp. 1095-1097, Nov. 1967.

[17] P. D. Lax, "Integrals of nonlinear equations of evolution and solitary waves," Commun. Pure Appl. Math, vol. 21, no. 5, pp. 467-490, Sep. 1968.

[18] V. E. Zakharov and F. Calogero, What is Integrability?, ser. Springer Series in Nonlinear Dynamics. Berlin, Germany: Springer-Verlag, 1991.

[19] A. C. Singer, "Signal processing and communication with solitons," Ph.D. dissertation, Dept. Electr. Eng., Massachusetts. Inst. of Technol., Cambridge, MA, USA, 1996.

[20] M. Reed and B. Simon, Methods of Modern Mathematical Physics: Vol 1, Functional Analysis. San Francisco, CA, USA: Academic Press, 1980.

[21] L. C. Evans, Partial Differential Equations, 2nd ed. Providence, RI, USA: Amer. Math. Soc., 2010.

[22] M. J. Ablowitz, D. J. Kaup, A. C. Newell, and H. Segur, "The inverse scattering transform - Fourier analysis for nonlinear problems," Stud. Appl. Math., vol. 53, no. 4, pp. 249-315, Dec. 1974.

[23] A. S. Fokas, "A unified transform method for solving linear and certain nonlinear PDE's," Proc. Roy. Soc. Lond. A, vol. 453, no. 1962, pp. 1411-1443, Jul. 1997.

[24] E. M. Stein and R. Shakarchi, Complex Analysis. Princeton, NJ, USA: Princeton University Press, 2003.

[25] M. Klaus and J. Shaw, "On the eigenvalues of Zakharov-Shabat systems," SIAM J. Math. Anal., vol. 34, no. 4, pp. 759-773, Feb. 2003.

[26] N. I. Muskhelishvili and J. R. M. Radok, Singular Integral Equations, 2nd ed. New York, NY, USA: Dover Publications, 2008. 
[27] M. J. Ablowitz and A. S. Fokas, Complex Variables: Introduction and Applications, 2nd ed. Cambridge, U.K.: Cambridge University Press, 2003.

[28] M. J. Ablowitz, B. Prinari, and A. D. Trubatch, Discrete and Continuous Nonlinear Schrödinger Systems, 1st ed., ser. Lond. Math. Soc. Lec. Note Series. Cambridge, U.K.: Cambridge University Press, 2003, vol. 302.

[29] T. Tao, Nonlinear Dispersive Equations: Local and Global Analysis, ser CBMS Regional Conf. Series in Math. Providence, RI, USA: Amer. Math. Soc., 2006, vol. 106.

Mansoor I. Yousefi received the Ph.D. degree from the University of Toronto and the M.Sc. degree (with honors) from Sharif University of Technology, Tehran, Iran, both in in Electrical Engineering. His research interests include information and communication theory, coding theory, optimization, optical communication systems and machine intelligence. He has won numerous awards and scholarships, including the IEEE Jack Keil Wolf ISIT Student Paper Award in 2013, the Edward S. Rogers Scholarship, the McAllister Graduate Fellowship, the Shahid U. H. Qureshi Prize and the J. L. Allen Yen Scholarship at the University of Toronto, and the Electrical Engineering Department Award at Sharif University of Technology.

Frank R. Kschischang received the B.A.Sc. degree (with honors) from the University of British Columbia, Vancouver, BC, Canada, in 1985 and the M.A.Sc. and Ph.D. degrees from the University of Toronto, Toronto, ON, Canada, in 1988 and 1991, respectively, all in electrical engineering. He is a Professor of Electrical and Computer Engineering at the University of Toronto, where he has been a faculty member since 1991. During 1997-98, he was a visiting scientist at MIT, Cambridge, MA; in 2005 he was a visiting professor at the ETH, Zurich, and in 2011 and again in 2012-13 he was a visiting Hans Fischer Senior Fellow at the Institute for Advanced Study at the Technical University of Munich.

His research interests are focused primarily on the area of channel coding techniques, applied to wireline, wireless and optical communication systems and networks. In 1999 he was a recipient of the Ontario Premier's Excellence Research Award and in 2001 (renewed in 2008) he was awarded the Tier I Canada Research Chair in Communication Algorithms at the University of Toronto. In 2010 he was awarded the Killam Research Fellowship by the Canada Council for the Arts. Jointly with Ralf Kötter he received the 2010 Communications Society and Information Theory Society Joint Paper Award. He is a recipient of the 2012 Canadian Award in Telecommunications Research. He is a Fellow of IEEE, of the Engineering Institute of Canada, and of the Royal Society of Canada.

During 1997-2000, he served as an Associate Editor for Coding Theory for the IEEE TRANS ACTIONS ON INFORMATION THEORY, and since January 2014, he serves as this journal's Editor-in-Chief. He also served as technical program co-chair for the 2004 IEEE International Symposium on Information Theory (ISIT), Chicago, and as general co-chair for ISIT 2008, Toronto. He served as the 2010 President of the IEEE Information Theory Society. 